\title{
The Role of High-Fat Diets in Exacerbating Cognitive Deficits After Traumatic Brain Injury
}

\author{
Michelle A. Frankot \\ West Virginia University, mf0083@mix.wvu.edu
}

Follow this and additional works at: https://researchrepository.wvu.edu/etd

Part of the Biological Psychology Commons

\section{Recommended Citation}

Frankot, Michelle A., "The Role of High-Fat Diets in Exacerbating Cognitive Deficits After Traumatic Brain Injury" (2020). Graduate Theses, Dissertations, and Problem Reports. 7759.

https://researchrepository.wvu.edu/etd/7759

This Thesis is protected by copyright and/or related rights. It has been brought to you by the The Research Repository @ WVU with permission from the rights-holder(s). You are free to use this Thesis in any way that is permitted by the copyright and related rights legislation that applies to your use. For other uses you must obtain permission from the rights-holder(s) directly, unless additional rights are indicated by a Creative Commons license in the record and/ or on the work itself. This Thesis has been accepted for inclusion in WVU Graduate Theses, Dissertations, and Problem Reports collection by an authorized administrator of The Research Repository @ WVU. For more information, please contact researchrepository@mail.wvu.edu. 
The Role of High-Fat Diets in Exacerbating Cognitive Deficits After Traumatic Brain Injury

\author{
Michelle A. Frankot
}

Thesis Submitted to the Eberly College of Arts and Sciences at West Virginia University

in partial fulfillment of the requirements for the degree of

Master of Science in

Psychology

\author{
Cole Vonder Haar, Ph.D., Chair \\ Steven Kinsey, Ph.D. \\ Rudolph Castellani, M.D. \\ Department of Psychology
}

Morgantown, West Virginia

2020

Keywords: traumatic brain injury; risky decision-making; high-fat diet; inflammation Copyright 2020 Michelle Frankot 


\begin{abstract}
The Role of High-Fat Diets in Exacerbating Cognitive Deficits After Traumatic Brain Injury
\end{abstract}

Michelle A. Frankot

Traumatic brain injury (TBI) can cause chronic psychiatric-like impairments that may be driven by inflammation in the brain. In the current study, inflammation was upregulated using a high-fat diet (HFD) to assess the role of inflammation in TBI-induced deficits. Rats were randomly assigned to receive an HFD or calorie-matched low-fat diet (LFD) for the duration of the experiment. After two weeks of free access to their respective diets, rats began behavioral training on the Rodent Gambling Task (RGT), during which they were allowed to freely choose to nosepoke in one of four holes in a standard operant chamber. Responses in each hole were associated with different probabilities and magnitudes of reinforcement (sucrose pellets) or punishment (timeout from reinforcement); thus, choices could be classified as either risky or optimal. Premature responses (i.e., nosepokes made before the trial began) were used as a measure of motor impulsivity. After behavior on the RGT stabilized, rats received either a frontal TBI or a sham procedure and continued post-injury testing for 10 weeks. TBI rats substantially decreased in optimal choice but increased in risky choices and motor impulsivity. However, deficits induced or exacerbated by the HFD were inconsistent and low in magnitude. After the behavioral portion of the study, rats were transcardially perfused. The HFD and TBI in combination interacted to increase neuroinflammation, as measured by microglia count. Increases in microglia unaccompanied by changes in behavior indicated that inflammation may simply be a symptom of brain injury and not a driver of psychiatric-like deficits. Thus, further evidence is required to characterize the role of inflammation in cognitive impairment both within and outside the context of brain injury. 


\section{Table of Contents}

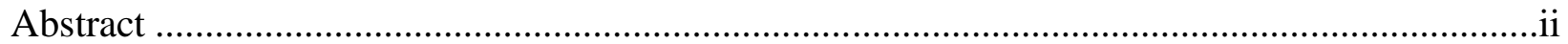

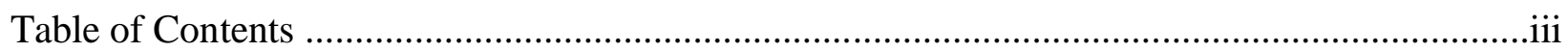

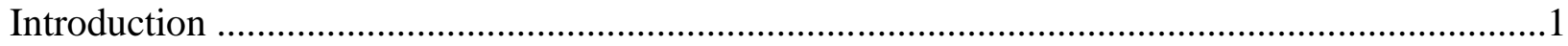

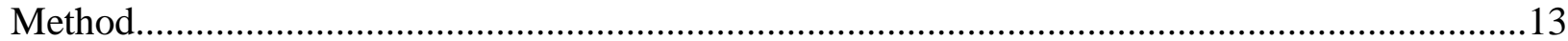

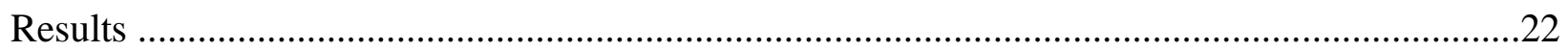

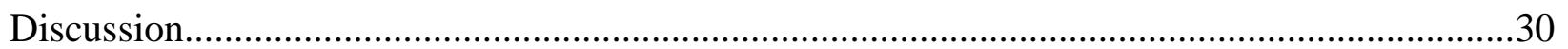

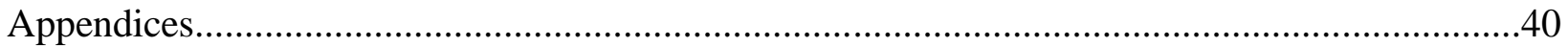

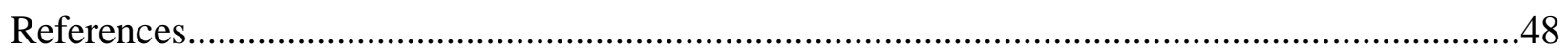




\section{INTRODUCTION}

Traumatic brain injury (TBI) can be defined as pathology of the brain resulting from external force (Menon, Schwab, Wright, \& Maas, 2010). Approximately 10 million people worldwide are annually affected by severe TBI, and it is one of the top 15 causes of death among individuals under 60 (Hyder, Wunderlich, Puvanachandra, Gururaj, \& Kobusingye, 2007), and a leading cause of death and disability among adolescents (Faul, $\mathrm{Xu}$, Wald, Coronado, \& Dellinger, 2010). However, even for individuals that physically recover after TBI, brain injuries are often associated with long term psychiatric symptoms and cognitive deficits (Zgaljardic et al., 2015). For example, following TBI, chronic impulsivity is often seen (Dixon et al., 2005; McHugh \& Wood, 2008; Zgaljardic et al., 2015) and may manifest itself in the form of substance abuse, sexual disinhibition, and aggression (McAllister, 1992).

Further, certain premorbid risk factors, such as age at time of injury (Garcia, Hungerford, \& Bagner, 2014; King, 2014), pre-injury family environment (Yeates et al., 1997), and lower socioeconomic status (Yeates et al., 2004) may predispose people to experience more severe deficits following TBI. A major potential risk factor that is underexplored is the role of diet in outcomes after TBI; it is currently unknown whether consumption of a high-fat diet may exacerbate chronic psychiatric-like deficits following TBI. Because it is difficult to experimentally identify causal effects of TBI with humans, preclinical animal models are often used. In the current study, a rat model of TBI was used to assess high-fat diet exposure as a risk factor for chronic deficits following TBI.

\section{Preclinical Models of Traumatic Brain Injury}

One common preclinical model of TBI is the controlled cortical impact (CCI) model developed by Lighthall (1988) to mechanically induce a focal TBI in ferrets. The CCI model is a 
surgical procedure in which a craniotomy is performed to expose the cortex. A metal, cylindrical impactor is used to contact the dura mater with mechanical force and induce a TBI (Lighthall, 1988). The CCI model was adapted for rats by Dixon, Clifton, Lighthall, Yaghmai, and Hayes (1991) and has become a useful method for the study of brain injury because it allows for experimental control of various parameters that affect the TBI (Dixon et al., 1991), such as the velocity, depth, size, and force of the impactor (Osier \& Dixon, 2016). The CCI model has been characterized as a clinically-relevant method for inducing consistent, moderate to severe contusions in animal models (Chen, Zhang, Lu, \& Wang, 2014). Further, single CCI injuries consistently result in deficits in learning and memory, as measured by the Morris Water Maze (Dixon et al., 1991; Longhi et al., 2004; Xiong et al., 2012), whereas other animal models of TBI, such as the fluid percussion injury, tend to result in acute, but not persistent, spatial deficits (Hamm, Pike, Temple, O'Dell, \& Lyeth, 1995; Shultz et al., 2012). As such, this model is ideal for the experimental study of chronic cognitive dysfunction following moderate to severe TBI.

Cognitive function is difficult to define, but it broadly involves the mental processes that allow for sensory input to be used to produce meaningful behavioral responses (Miller \& Wallis, 2009). Although cognitive processes are associated with widely distributed areas of the brain, several "higher-order" (i.e., requiring integration across multiple sensory modalities to produce appropriate motor output) processes that are impaired in various psychiatric conditions, such as decision-making and impulse control, are specifically associated with the prefrontal cortex in clinical and preclinical populations (Winstanley, Eagle, \& Robbins, 2006). Thus, the CCI model can be applied to the prefrontal cortex to study complex cognitive processes that are associated with overt, psychiatrically-relevant behaviors. 


\section{Cognitive Deficits after Traumatic Brain Injury}

\section{Impulsivity in Clinical Populations}

One particularly important cognitive deficit that can accompany frontal brain dysfunction is impulsivity. Impulsivity, or actions that facilitate short-term reinforcement at the expense of long-term outcomes, is a key symptom of at least 20 different diagnostic criteria of the Diagnostic and Statistical Manual of Mental Disorders (American PsychiatricAssociation, 2013; Griffin, Lynam, \& Samuel, 2018). According to a recent meta-analysis, impulsive behavior is a core feature in a variety of both substance and behavioral addictions (Lee, Hoppenbrouwers, \& Franken, 2019). Preclinical TBI research tends to be dominated by hippocampal-dependent measures. However, it is important to study the underlying mechanisms of other psychiatric symptoms that contribute to numerous disorders and impact daily functioning at the clinical level. Impulsivity in the context of TBI is of particular importance to daily function because it can contribute to aggression, disinhibition, and inattention (Vaishnavi, Rao, \& Fann, 2009).

Impulsivity after TBI can be further subdivided into two categories: motor and choice impulsivity. Motor impulsivity, or behavioral disinhibition, involves the inability to inhibit actions, whereas the choice component involves making decisions with a disregard for long-term consequences (Ozga, Povroznik, Engler-Chiurazzi, \& Vonder Haar, 2018; Winstanley et al., 2006). Motor impulsivity is assessed in humans using tasks that involve one stimulus that signals responses will be reinforced and another stimulus that signals response inhibition will be reinforced (e.g., Go/No-Go task). In multiple studies using this type of assessment, individuals with a moderate to severe TBI had poorer response inhibition than matched controls (Nativ, Lazarus, Nativ, \& Joseph, 1994; Rochat, Beni, Annoni, Vuadens, \& Van der Linden, 2013). 
Motor impulsivity following TBI in humans is of particular importance because it is associated with impulsive aggression (Alderman, 2003; Dyer, Bell, McCann, \& Rauch, 2006).

\section{Impulsivity in Preclinical Populations}

There have been recent efforts to study the effects of TBI on chronic motor impulsivity. One method that is often used to study motor impulsivity in rats is the five-choice serial reaction time (5-CSRT) task. In this task, a brief light flashes in one of five holes in an operant chamber. Rats must nosepoke in the correct hole to receive a reinforcer and withhold premature responses to avoid timeouts from reinforcement (Carli, Robbins, Evenden, \& Everitt, 1983). Even mild TBI impairs motor impulse control as seen by increases in premature responding on the 5-CSRT (Vonder Haar et al., 2016). Go/No-Go tasks (similar to those used with humans) can also be incorporated into the 5-CSRT. In this version of the Go/No-Go paradigm, a single stimulus light functioned as a "go" signal to indicate that responses would be reinforced. The illumination of all five lights functions as a "no-go" signal to indicate that only a lack of response will be reinforced. In rodents, mild TBI caused an inability to withhold responses on the Go/No-Go paradigm of the 5-CSRT (Hehar, Yeates, Kolb, Esser, \& Mychasiuk, 2015; Mychasiuk, Hehar, \& Esser, 2015).

However, impulsivity is one of many deficits that emerges following TBI. Specifically, TBI-induced motor impulsivity may be closely tied to risky decision-making. A meta-analysis indicated that motor impulsivity was positively correlated with risky decision-making in healthy male rats (Barrus, Hosking, Zeeb, Tremblay, \& Winstanley, 2015). There is also preliminary data that suggests a connection between risky decision-making and impulsivity in human subjects (Chase et al., 2017). Thus, it is important to assess risky decision-making following TBI 
in addition to motor impulsivity to model the types of detrimental choices that may results in poor outcomes for TBI patients.

\section{Risky Decision-Making in Clinical Populations}

Risky decision-making shares many commonalities with impulsive choice but differs in one key element: impulsive choices always result in a poor outcome, whereas risky decisions probably result in a poor outcome (Ozga et al., 2018). The Iowa Gambling Task (IGT) is a card game used to assess risky decision-making in clinical populations (Bechara, Damasio, Damasio, \& Anderson, 1994). The game targets decision-making with uncertain of outcomes and involves a choice between short- and long-term monetary pay offs. During the IGT, subjects are given a mock currency and required to repeatedly pick a card from one of four separate decks. After choosing a card, subjects are given currency (i.e., gains) but they may also be required to give up some currency (i.e., losses). The decks are associated with different magnitudes of gains and losses. The decks associated with large gains are also associated with large losses; choices from these decks do not result in the highest net gains and are considered "risky" decisions. The decks associated with smaller gains are also associated with smaller losses; choices from these decks are considered optimal because they result in the largest net gain over time. Patients with prefrontal cortex damage received lower net gains over time because they made risky decisions based on immediate consequences (i.e., large immediate gains) rather than overall future outcomes (Bechara et al., 1994). Performance on the IGT is often impaired in humans with frontal brain pathologies (e.g., large frontal lesions, ventromedial prefrontal cortex damage) due to various conditions, such as aneurysms, cysts, and surgical brain tumor removal (Manes et al., 2002; Waters-Wood, Xiao, Denburg, Hernandez, \& Bechara, 2012; Zinchenko \& Enikolopova, 
2017). However, experimental animal models are needed to determine causal effects of frontal injuries on risky decision-making.

\section{Risky Decision-Making in Preclinical Populations}

The Rodent Gambling Task. The Rodent Gambling Task, a rat analogue of the Iowa Gambling Task, assesses decision-making with uncertain outcomes (van den Bos, Lasthuis, den Heijer, van der Harst, \& Spruijt, 2006). In the early version of this task, rats were able to choose between four arms connected to their chamber to earn a sucrose reinforcer. Two arms occasionally contained a large reinforcer among unpalatable quinine pellets, and the others consistently contained a small reinforcer among the quinine pellets. The arms that contained the small reinforcer were considered optimal because they delivered the highest probability of reinforcement in the long-term (van den Bos et al., 2006). Several years of research using this Rodent Gambling Task repeatedly implicated the frontal cortex in making optimal long-term decisions for small reinforcers, rather than risky choices for large reinforcers that may be accompanied by punishment (van den Bos, Koot, \& de Visser, 2014).

More recently, the task was adapted to include punishments that more closely reflect the IGT and capture the risk of losing in addition to the absence of gain (Zeeb \& Winstanley, 2013). In this modified Rodent Gambling Task (which will now be referred to as the RGT), rats choose between four options by nose-poking in one of four holes in a standard operant chamber. Each hole is associated with a different number of reinforcers (sucrose pellets) and a different probability of reinforcer delivery. Rats must also withhold responses during a 5-second delay, which provides a measure of motor impulsivity. The choices that occasionally provide larger reinforcers are also associated with a high probability of timeout from reinforcement, or a duration during which responses never yield reinforcement. These timeouts decrease overall 
reinforcement rate, making the larger reinforcer options less optimal in the long-term. Healthy control rats choose the optimal option most frequently, followed by the riskier options (Zeeb \& Winstanley, 2013), but TBI rats have persistent reductions in optimal choice for 12 weeks postinjury. Further, TBI increases risky choice and motor impulsivity (Shaver et al., 2019). Overall, the RGT appears to be an effective task for capturing chronic cognitive deficits following TBI in rats given that it encompasses both risky decision-making and motor impulsivity. This task can also be used to assess risk factors that affect the course of behavior after TBI.

\section{Risk Factors for Chronic Deficits After TBI}

There is considerable variability in symptoms and deficits after brain injury in humans. For some individuals, symptoms may resolve within days to weeks, whereas other may have symptoms that persist for years (Dwyer \& Katz, 2018). Risk factors for persisting symptoms include multiple injuries (Guskiewicz et al., 2003), pre-existing psychiatric symptoms (Cooper et al., 2011; King, 2014; King \& Kirwilliam, 2011; Mooney \& Speed, 2001), age at time of injury (Garcia et al., 2014; King, 2014), and pre-injury family environment (Yeates et al., 1997). Additionally, lower socioeconomic status (SES) is predictive of poorer outcomes following TBI (Yeates et al., 2004). SES is a broad construct that is traditionally defined by education, income, and occupation. Each of these variables can affect health-related outcomes within a household, including recreation, access to health care, mortality, living conditions, and nutrition (Adler \& Newman, 2002). It is plausible that nutrition partially explains the relationship between SES and recovery after TBI, given that low SES is associated with poor diet quality (Pechey \& Monsivais, 2016; Shahar, Shai, Vardi, Shahar, \& Fraser, 2005), and particularly increased fat intake compared to high SES individuals (Darmon \& Drewnowski, 2008; Giskes, Avendano, Brug, \& 
Kunst, 2010; Shimakawa et al., 1994). Taken together, these studies suggest high-fat diets (HFD) could be explored as a potential premorbid risk factor for poorer outcomes following TBI.

\section{High-Fat Diets}

An HFD is calorically dense food that is primarily used to induce obesity in rodent models. This diet-induced obesity (DIO) model has enabled the experimental study of the effects of a Western-type diet, which tends to be high in fat, and causes weight gain as a result (Cordain et al., 2005; Fung et al., 2001). However, HFD consumption has a number of effects in addition to causing weight gain and overeating (Hariri \& Thibault, 2010). For example, HFD consumption, like TBI, can cause neuroinflammation and cognitive deficits (Beilharz, Maniam, \& Morris, 2015; Miller \& Spencer, 2014). Greenwood and Winocur (1990) first demonstrated that an HFD caused deficits in learning and memory. Compared to rats fed a control diet, HFDfed rats were impaired on a radial arm maze (measure of hippocampal-dependent spatial memory), a variable-interval delayed alternation task (measure of frontally-mediated rulelearning), and the Hebb-Williams maze series (measure of general cognitive function).

In a more recent experiment, acute presentation (72 hr free access) of an HFD caused deficits in spatial memory, while long-term presentation (30 days) caused deficits in non-spatial working memory (Kanoski \& Davidson, 2010). There are also recent experiments that demonstrate the effects of an HFD on operant tasks that capture chronic non-spatial cognitive deficits, such as impulsivity. For instance, rats maintained on a chronic HFD had more premature responding on the 5-CSRT than rats maintained on a matched low-fat diet (LFD). This increase in premature responding is indicative of motor impulsivity and did not occur for rats maintained on a high-sugar diet. Thus, increased impulsivity was attributed to the fat content of the diet (Adams et al., 2015) Similarly, rats maintained on an HFD for 8 weeks made more impulsive 
choices on a delay discounting task, compared to control rats (Steele, Pirkle, \& Kirkpatrick, 2017). Taken together, these findings show that HFD consumption can impair behavior on a variety of tasks. The mechanism driving this behavioral change may be neuroinflammation (to be discussed further below), a symptom affected by brain injury as well as diet.

\section{Diet and TBI}

There are relatively few studies that examine diet in conjunction with brain injury. However, dietary manipulations may have a particularly pronounced effect on recovery from TBI given that metabolic dysfunction occurs as a result of brain injury (Gasco et al., 2012; Prodam et al., 2013; Ziablitsev, Pishchulina, Kolesnikova, Boris, \& Yuzkiv Ya, 2016). Existing literature suggests that certain diets, specifically HFDs, may exacerbate the symptoms of TBI. For example, 8-week maintenance on an HFD worsened the recovery of sensorimotor and working memory deficits following CCI in rats (Hoane, Swan, \& Heck, 2011). Similarly, rats given a mild pediatric concussion and HFD experienced more severe deficits in balance and exploration, compared to control-fed rats with a concussion (Mychasiuk, Hehar, Ma, \& Esser, 2015). Although very little is known about how HFD exposure may specifically affect chronic cognitive dysfunction resulting from TBI, it appears that HFD exposure can alter the course of recovery following TBI. In order to assess the additive effects of TBI and HFD exposure, neuroinflammation, an area where symptoms of the two overlap, must be considered.

\section{Psychiatric Disease and Inflammation}

Inflammation is the body's innate and adaptive response to disruptions to homeostasis (e.g., infection, physical injury) that involves transportation of blood components, release of inflammatory mediators, and the release of toxins to destroy pathogens (Medzhitov, 2008). In the short term, this is a beneficial adaptation but can become detrimental when activated chronically. 
Chronic inflammation is a characteristic comorbidity of a number of psychiatric diseases and symptoms. Specifically, neuroinflammation in humans has been linked to impulsivity, suicidality, bipolar disorder, and schizophrenia (Isung et al., 2014; Najjar, Pearlman, Alper, Najjar, \& Devinsky, 2013). These psychiatric symptoms are often associated with TBI as well. In particular, TBI has high comorbidities with psychiatric disorders involving impulse control, such as various substance use disorders (Koponen et al., 2002; Whelan-Goodinson, Ponsford, Schönberger, \& Johnston, 2010). A chronic neuroinflammatory response following TBI has been characterized in humans (Gentleman et al., 2004) and has been identified as a potential variable mediating the relationship between TBI and chronic psychiatric-like symptoms in rats (Vonder Haar et al., 2016). In the current study, we assessed the interaction between HFD and TBI and hypothesized that the two in conjunction would amplify inflammation-induced behavioral impairments.

\section{Mechanisms of TBI and Diet Relationship}

\section{Neuroinflammation}

Clinical literature. There is evidence to suggest that TBI and HFD exposure may have additive dysregulatory effects mediated by neuroinflammation. In humans, TBI has been thoroughly linked to neuroinflammation (for a review, see Jassam, Izzy, Whalen, McGavern, \& El Khoury, 2017; Simon et al., 2017). The literature linking neuroinflammation with HFDs in clinical populations is somewhat sparse, but there is evidence to suggest the two are related. For example, increased levels of circulating lipopolysaccharide-binding protein, a neuroinflammatory marker associated with HFD consumption (Moreno-Navarrete et al., 2012), have been linked with poorer performance on cognitive working memory tasks in humans (Moreno-Navarrete et al., 2017). Although obesity has been linked to increased 
neuroinflammation (Guillemot-Legris \& Muccioli, 2017; Miller \& Spencer, 2014), experimental DIO models are needed to directly connect HFD exposure with neuroinflammation.

Preclinical literature. As previously reviewed, HFD exposure causes neuroinflammation and accompanying deficits in cognition in experimental animal models (Beilharz et al., 2015; Greenwood \& Winocur, 1990; Hoane et al., 2011; Kanoski \& Davidson, 2010; Ledreux, Wang, Schultzberg, Granholm, \& Freeman, 2016; Miller \& Spencer, 2014; Mychasiuk, Hehar, Ma, et al., 2015). Brain injury-induced neuroinflammation is often linked to psychiatric and cognitive deficits in rats, such as impulsive choice and motor impulsivity (Vonder Haar et al., 2016; Vonder Haar et al., 2017). Given that neuroinflammation is caused by both TBI (Chiu et al., 2016; Simon et al., 2017) and HFD consumption (Miller \& Spencer, 2014), brain injury after chronic HFD exposure may have an additive effect on impaired cognition.

Markers of inflammation. Inflammation can be characterized in the brain by quantifying cells involved in the inflammatory response such as microglia morphology. Microglia are cells of the nervous system responsible for surveying the CNS and initiating immune responses (Harry, 2013). In response to injury, acute microglia activation can have reparatory effects, but chronic activation, as seen after TBI, stimulates the release of proinflammatory cytokines, including interleukin (IL)-1 $\beta$, IL-6, and tumor necrosis factor- $\alpha$ (Donat, Scott, Gentleman, \& Sastre, 2017; Smith, Das, Ray, \& Banik, 2012). Increases in microglia expression have been experimentally induced by traumatic brain injury (Fenn et al., 2014; Karelina, Nicholson, \& Weil, 2018; Muccigrosso et al., 2016) and HFD exposure (Jeon et al., 2012; Ledreux et al., 2016; Pepping, Freeman, Gupta, Keller, \& Bruce-Keller, 2013). Thus, the role of neuroinflammation in mediating cognitive deficits resulting from TBI and HFD consumption can be assessed by quantifying microglia. 


\section{The Current Study}

Currently, the effects of HFD exposure on the long-term recovery of cognitive and behavioral function after TBI remain unclear. In the current study, HFD exposure was assessed as a risk factor for chronic risky decision-making following TBI. Male rats were given access to either an HFD or LFD and trained on the RGT. After behavior was stable on the RGT, rats were assigned to receive a TBI or a sham injury. It was predicted that TBI rats with HFD exposure would have the most severe impairments in decision-making, as operationalized by a reduction in optimal choice or increase in risky choice on the RGT. It was also predicted that TBI rats with HFD exposure would have the most severe motor impulsivity, as operationalized by an increase in premature responding on the RGT. The proposed mechanism driving these TBI and HFDinduced deficits was neuroinflammation. Thus, it was predicted that TBI rats with HFD exposure would have the highest activation of microglia, a sign of the inflammatory response. 


\section{METHOD}

\section{Animals and Diets}

Subjects were adult male Long-Evans rats $(N=40)$ obtained from Charles River at approximately $250-275 \mathrm{~g}$ body weight as determined by a priori power analysis. However, 4 rats had to be euthanized prior to the end of the experiment due to poor recovery from surgery. Rats were pair-housed in OptiRAT cages (Animal Care Systems, Centennial, CO) with a divider separating the two animals and maintained on a 12:12 reverse light-dark cycle with water available ad libitum. Prior to beginning the experiment, rats were pseudorandomly assigned (for practicality, all rats sharing a cage received the same diet type) to receive either a high-fat or low-fat diet matched for sugar and other nutrient contents. The HFD consisted of $60 \% \mathrm{kcal}$ from fat (Research Diets; D12492; $5.2 \mathrm{kcal} / \mathrm{g}$ ), and the LFD consisted of 10\% kcal from fat (Research Diets; D12450J; $3.8 \mathrm{kcal} / \mathrm{g}$ ). For two weeks, rats were given ad libitum access to their respective diets. Then, rats were food restricted to $\sim 85 \%$ free feeding weight to increase motivation for sucrose reinforcers in operant tasks. Food restriction occurred gradually over 2 weeks, tapering from $70 \mathrm{kcal}$ to $48 \mathrm{kcal}$ per day. Rats were then maintained on a $48 \mathrm{kcal} /$ day diet that matched the physiological fuel value of $14 \mathrm{~g}$ per day of a control diet $(3.1 \mathrm{kcal} / \mathrm{g})$. This food regimen was based on published data (Adams et al., 2015). Rats were weighed twice per week to ensure that the diet manipulation did not differentially affect body weight. All procedures (denoted in a timeline in Figure 1) were approved by the West Virginia University Institutional Animal Care and Use Committee prior to the start of any experiments. 


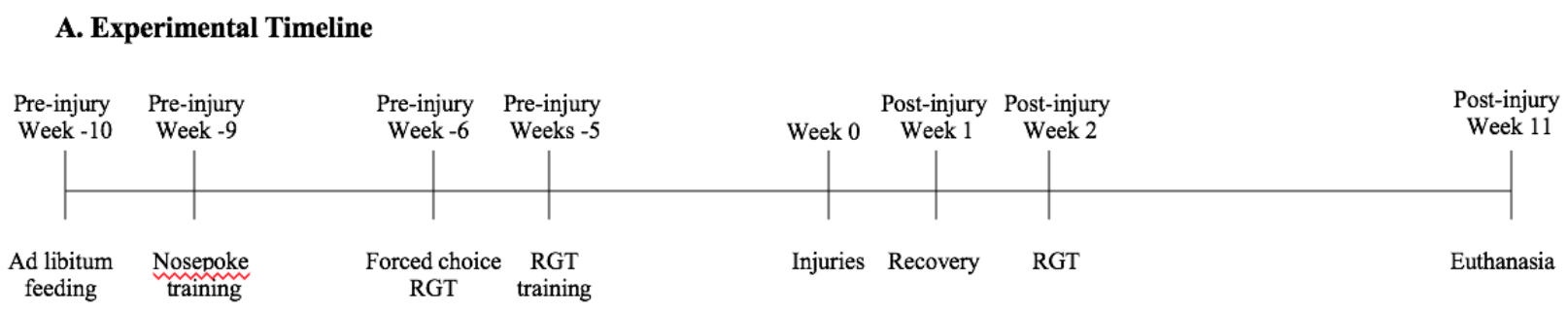

Figure 1. The experimental timeline. Negative week numbers represent weeks prior to injury; positive week numbers represent weeks after injury. Rats were free fed a high-fat (HFD) or lowfat diet (LFD). Then, they were trained on an operant gambling task. When behavior was stable, bilateral frontal injuries were induced, and testing continued for 10 weeks. Rats were euthanized during post-injury Week 11, and brain tissue was collected.

\section{Apparatus}

Behavioral testing was conducted in a set of 16 operant chambers. Each chamber was enclosed in a sound-attenuating box, and white noise $(\sim 70 \mathrm{~dB})$ was played in the room to ensure that each rat was exposed to the same auditory environment. The floor of each chamber was composed of a parallel stainless-steel rods. The right side of each chamber was equipped with a food hopper and associated light. Retractable levers with cue lights were situated on each side of the food hopper. The levers were never extended during the course of the experiment. The left wall of the chamber was equipped with a 5-hole array in which the nosepokes were recorded. The chamber was also equipped with a houselight and tone generator. Only the 5-hole array (and its associated lights), the food hopper, hopper light, and the houselight were used during behavioral training and testing.

\section{Behavioral Training}

One day prior to exposure to the operant chambers, each rat was given approximately 10 sucrose pellets in their home cage. The next two days, rats habituated to the operant chambers for 30 min. During habituation, several sucrose pellets were placed in each hole as well as the food 
hopper. Then, the behavior of nosepoking in the holes was shaped using the training regimen for the 5-CSRT (Carli et al., 1983). A stimulus light was illuminated in a single hole for $30 \mathrm{~s}$; a response in the illuminated hole (i.e., a nosepoke) resulted in reinforcement (i.e., the delivery of a sucrose pellet). As performance improved, the duration of the stimulus light was gradually titrated down to 10 s. Premature responses (i.e., before illumination of the stimulus light), omissions, and incorrect responses were punished with a 5-s timeout from reinforcement. Training continued for 17 sessions until a minimum of $80 \%$ accuracy was achieved and fewer than $20 \%$ trials were omitted.

\section{Rodent Gambling Task Training}

In the RGT, four options (i.e., four holes) were presented in each trial. The one-pellet choice (P1) has a $90 \%$ probability of reinforcement and a $10 \%$ probability of a 5 -s timeout from reinforcement. The P1 option was considered non-risky, but suboptimal because it did not deliver the highest overall rate of reinforcement. The two-pellet choice (P2) had an $80 \%$ probability of reinforcement and a $20 \%$ probability of a 10 -s timeout from reinforcement. P2 was considered the optimal choice, because it delivered the highest overall rate of reinforcement over the course of a full session. The three-pellet (P3) and four-pellet (P4) options were both considered risky, suboptimal choices because they delivered the lowest overall rates of reinforcement. The $\mathrm{P} 3$ option had a $50 \%$ probability of reinforcement and a $50 \%$ probability of a 30 -s timeout; the $\mathrm{P} 4$ option had a $40 \%$ probability of reinforcement and a $60 \%$ probability of a 40-s timeout. During the timeout, no responses were reinforced, and the light in the previouslychosen hole slowly flashed for the duration $(1 \mathrm{~Hz})$. A schematic of the task can be seen in Figure 2 (Shaver et al., 2019). The location of the P1, P2, P3, and P4 holes were counterbalanced across animals to account for potential side bias. 


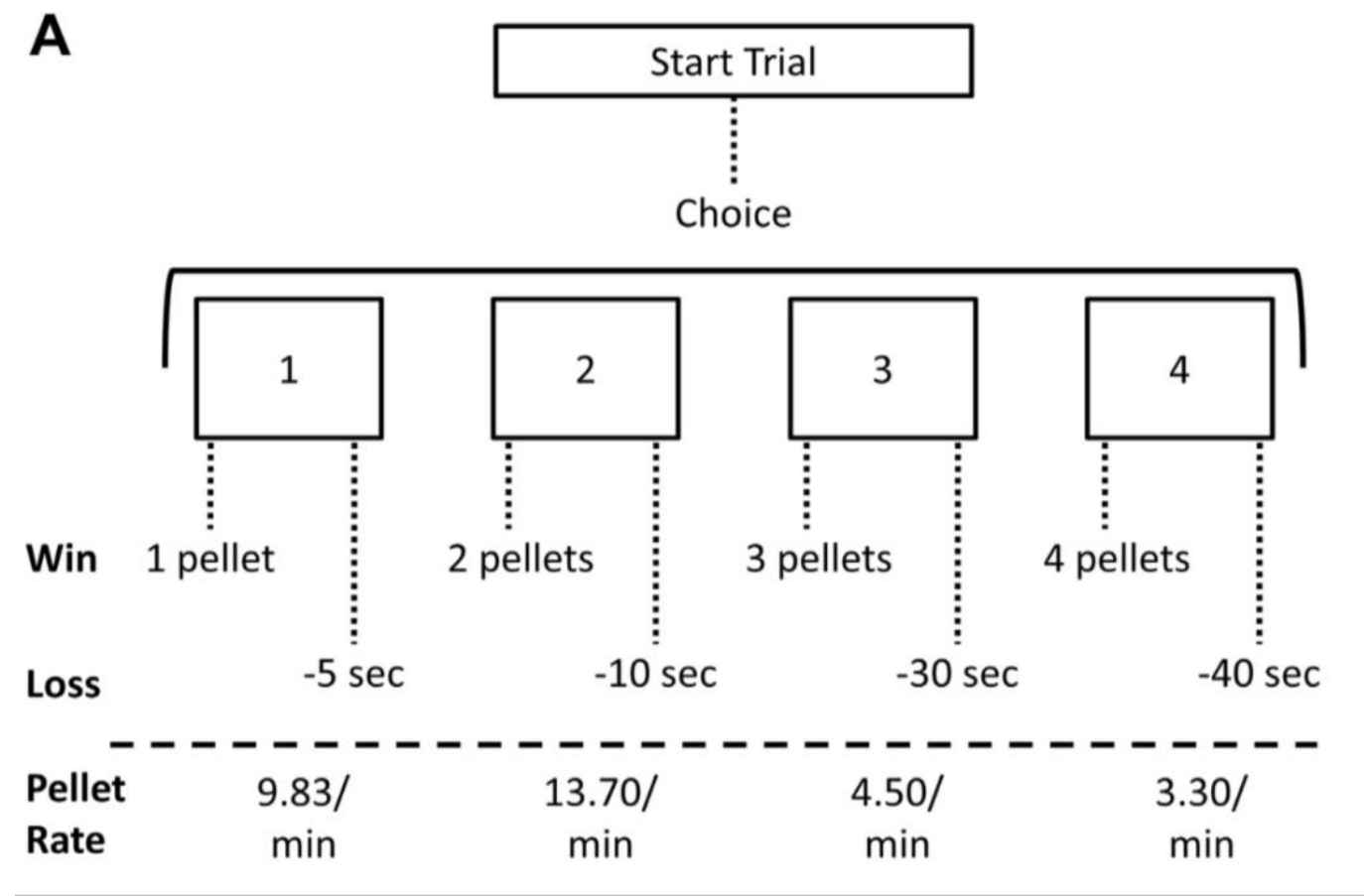

Figure 2. A schematic of the Rodent Gambling Task (RGT). After initiating a trial, rats were able to choose from any of the four holes. Each hole was associated with a different probability and magnitude of reinforcement and punishment (Shaver et al., 2019).

All rats began with a forced-choice training procedure. This procedure was identical to the full RGT task, but only one choice was presented at a time. This ensured that the four choice options were experienced equally. Seven sessions of forced-choice RGT were conducted before progressing to the full task. During the full task, rats were able to freely choose among any of the four choices.

The full RGT task consisted of daily 30-min sessions with a maximum of 250 trials per session. Prior to each trial, the light above the food hopper was illuminated. A nosepoke in the food hopper in response to the light initiated a trial, and a 5-s intertrial interval (ITI) began. Any nosepokes during the ITI were categorized as a premature response and resulted in a 5-s illumination of the houselight. Following the 5-s ITI, stimulus lights above holes 1, 2, 4, and 5 (corresponding to choices $\mathrm{P} 1, \mathrm{P} 4, \mathrm{P} 2, \mathrm{P} 3$ in one version, or $\mathrm{P} 4, \mathrm{P} 1, \mathrm{P} 3, \mathrm{P} 2$ in the other) of the 5hole array were illuminated. Responses were then made freely in any one of the four holes. A 
response in any hole turned off the stimulus lights and resulted in either reinforcement (sucrose pellets) or punishment (timeout from reinforcement) according to the probabilistic schedule described above. Failure to respond within $10 \mathrm{~s}$ was defined as an omission. Rats were tested on the RGT five times per week for 5 weeks when behavior was stable. Baseline stability was defined as no effect of session on behavior over a 3-session within-subject analysis and confirmed by visual analysis of individual subjects. After all rats met baseline stability criteria, they were matched on RGT performance (using the P2 optimal choice variable) to prevent preinjury group differences and assigned to receive either a TBI or a sham injury.

\section{Surgery}

After meeting baseline stability on the RGT, rats were matched on optimal choice of the P2 option and then randomly assigned to receive a either a severe bilateral frontal TBI or a sham injury. A controlled cortical impact procedure was used to administer severe, focal TBIs. All rats were anesthetized with isoflurane (5\% induction, $2-4 \%$ maintenance) in $0.5 \mathrm{~L} / \mathrm{min}$ oxygen. Then rats were placed into a stereotaxic frame and administered a local analgesic (Bupivacaine; $0.25 \%$, s.c.) at the incision site and a subcutaneous general analgesic (ketoprofen; $5 \mathrm{mg} / \mathrm{kg}$, s.c.). Then, the surgical site was sanitized, and rats were given a midline incision. At this point, sham rats had the incision sutured shut after bleeding stopped, and the surgery was considered to be complete. This intact sham procedure is recommended to avoid potential confounds introduced by craniotomy drilling (Cole et al., 2011). For TBI rats, a $6 \mathrm{~mm}$, circular craniotomy centered at $\mathrm{AP}+3.0, \mathrm{ML}+0.0 \mathrm{~mm}$ from bregma was performed with a surgical drill to expose the frontal cortex. A Leica Impact One device (Leica Biosystems, Buffalo Grove, IL) was used induce a bilateral TBI with a consistent depth $(-2.5 \mathrm{~mm})$, velocity $(3 \mathrm{~m} / \mathrm{s})$, and dwell $(500 \mathrm{~ms})$. Following the impact, sterile gauze was used to stop the bleeding, and then the incision site was sutured 
shut. To prevent infection, triple antibiotic ointment was applied to the incision site of all rats (both TBI and sham).

\section{Behavior Assessment}

Rats were given seven days of post-surgical monitoring to recover. Following recovery, they resumed daily sessions of the RGT for 10 weeks post-injury. The primary variables of interest on the RGT were optimal decision-making (P2 choice), risky decision-making (P3 and P4 choice), and motor impulsivity (premature responses). Psychomotor variables, such as choice latency and reinforcer collection latency were also measured to capture general neurologic deficits.

\section{Immunohistochemistry}

During post-injury Week 11, rats were transcardially perfused with $0.9 \%$ phosphate buffered saline followed by $3.7 \%$ phosphate buffered formaldehyde. Brains were fixed in $3.7 \%$ phosphate buffered formaldehyde for $24 \mathrm{hr}$ before being transferred to a $30 \%$ sucrose solution. Brains were embedded in a gelatin block (15\% gelatin) with a maximum of four brains per block. The gelatin blocks were frozen $\left(-80^{\circ} \mathrm{C}\right)$ and then sliced using a sliding microtome at a thickness of $30 \mu \mathrm{m}$.

A stain for IBA-1, a microglia-specific protein in the rat brain (Ito et al., 1998) was performed to detect and quantify microglia. Tissue was blocked in $2 \%$ normal goat serum for 4$24 \mathrm{hr}$ at $4^{\circ} \mathrm{C}$. Then, the tissue was incubated with rabbit anti-IBA-1 primary antibody (WAKO 019-19741; $1: 2000)$ for $48 \mathrm{hr}$ at $4^{\circ} \mathrm{C}$, rinsed, and then incubated with goat anti-rabbit IgG secondary antibody (Vector BA-1000, 1:2000) for 90 min at room temperature. Tissue was rinsed and reacted with an avidin-biotin complex kit (Vectastain PK-6100) and catalyzed with $0.05 \%$ diaminobenzadine and $0.15 \%$ hydrogen peroxide at room temperature. Quantification of 
IBA-1 was conducted at three regions of interest: the perilesion area, hippocampus, and orbitofrontal cortex (OFC). The perilesion area was assessed to measure the effects of TBI on microglia, whereas the hippocampus served as a general measure of inflammation that has been implicated as a target of HFD-induced microglia expression (Jeon et al., 2012; Ledreux et al., 2016) due to its highly plastic response to manipulations (Anand \& Dhikav, 2012). The HPC was also used to control for the effects of tissue damage on inflammation; it is distal to the frontal lesion and should be relatively unaffected by the focal CCI injury. The OFC was assessed due to its heavy involvement in normal decision-making on the RGT (Zeeb \& Winstanley, 2013). Because the OFC is proximal to the injury but the tissue itself should remain intact, it was an ideal location to assess the interactive effects of injury and diet on RGT performance. Images of each region of interest were taken at 40x magnification using an Olympus BX43 microscope with DP-80 13.5 megapixel camera in CellSens software. Microglia cells were then counted by hand to average across group and region.

\section{Lesion Analysis}

Slices of the brain across the area of the lesion $(+5.0,+4.0,+3.0,+2.0,+1.0$ from bregma) (Paxinos \& Watson, 1982) were mounted onto charged slides that improved cohesion to the brain slices. Slides were rehydrated using the following washes: Citrisolv (1 x 10 min), 95\% EtOH (1 x $3 \mathrm{~min}), 70 \%$, EtOH (1 x $1 \mathrm{~min}), 50 \% \mathrm{EtOH}$ (1 x $1 \mathrm{~min})$, and dH2O (1 x 2 min). They were then placed in thionin (10 s) for staining and dehydrated by reversing the previous sequence of washes. After dehydration, slides were cover-slipped and allowed to dry overnight. Images of each slice (600 dpi) were measured using ImageJ (NIH, Bethseda, MD). Remaining brain volume and lesion size were quantified by multiplying the thickness $(30 \mu \mathrm{m})$ by distance and average slice area (Vonder Haar, Anderson, \& Hoane, 2011). 


\section{Data Analysis}

The effects of injury and diet on percent choice of each option in the RGT were determined by linear mixed effects regression. Predictors included week, injury (TBI vs. sham), diet (HFD vs. LFD), and choice. When a week x injury x diet x choice interaction was observed, the data for each individual choice (i.e., P1, P2, P3, P4) was assessed separately. The effects of the experimental manipulations on number of premature responses, omissions, and psychomotor variables were also assessed. IBA-1 levels were compared using two-way ANOVAs (injury x diet) for each region of interest.

Transformations were applied to non-normal distributions. An arcsine-squareroot transformation was applied to percent choice data, because these data were both upper and lower bound. Other variables, such as premature responses and omissions were positively skewed and required a log transformation. Inter-rater reliability for IBA-1 cell counts and lesion analysis was confirmed by a second blind rater and tested using a Pearson correlation and a paired samples $t$ test. The second rater checked 16 brains (2-4 images per brain) for cell counts and 6 brains ( 5 images per brain) for lesion analysis. For all analyses, statistical significance was determined by a priori criteria of $\alpha=.05$.

\section{Predictions/Hypotheses}

The four groups in the experiment were as follows: TBI/HFD, TBI/LFD, sham/HFD, sham/LFD. It was hypothesized that (1), TBI animals would increase in risky decision-making, motor impulsivity, and neuroinflammation. This potential main effect of TBI would be a replication of previous findings (e.g., Shaver et al., 2019). It was hypothesized that (2) HFD would have a similar effect; HFD consumption would also increase risky decision-making, motor impulsivity, and neuroinflammation. Lastly, it was hypothesized that (3) TBI and HFD 
would interact to produce the most robust deficits in risky decision-making, motor impulsivity, and inflammation. Risky decision-making and motor impulsivity were assessed using the RGT. It is important to note that deficits in decision-making deficits may manifest itself in a number of different ways (i.e., increased choice of risky options and/or decreased choice of optimal options). Motor impulsivity was assessed using the number of premature responses in the RGT. Inflammation was assessed by quantifying microglia in the brain regions of interest. 


\section{RESULTS}

The major findings from behavioral testing (pre and post-injury) and histology are provided below. Expanded statistics, including null findings, beta coefficients, and $t$-test statistics, can be found in tables (see Appendices). All beta coefficients were standardized.

\section{Pre-Injury RGT Choice}

P1 Choice. Pre-injury choice of the suboptimal P1 option (Table 1; Figure 3a) decreased across weeks of training $(p<0.001)$ but was unaffected by diet $(p=0.137)$.

P2 Choice. Pre-injury choice of the optimal P2 option (Table 1; Figure 3b) increased across weeks of training $(p<0.001)$ but was also unaffected by diet $(p=0.062)$.

P3 Choice. Pre-injury choice of the risky P3 option (Table 1; Figure 3c) did not change across weeks $(p=0.477)$ and was unaffected by diet $(p=0.219)$.

P4 Choice. Pre-injury choice of the risky P4 option (Table 1; Figure 3d) did not change across weeks $(p=0.219)$, but the HFD rats made fewer P4 choices than the LFD rats $(p<0.001)$.

\section{Pre-Injury RGT Premature Responses, Omissions, and Reinforcers}

Premature responses. Pre-injury premature responses (Table 2; Figure 4a) decreased across weeks of training $(p<0.001)$ but were unaffected by diet $(p=0.429)$.

Omissions. Pre-injury omissions (Table 2; Figure 4b) decreased across weeks of training for HFD rats only $(p=0.044)$.

Reinforcers. Reinforcers earned during the RGT (Table 2; Figure 4c) increased across weeks of training $(p<0.001)$ but were unaffected by diet $(p=0.943)$.

\section{Pre-Injury RGT Psychomotor Variables}

Choice latency. Latency to choose a response (Table 3; Figure 5a) decreased across weeks of training $(p=0.027)$, and this decrease was most pronounced for HFD rats $(p<0.001)$. 
Collection latency. Latency to collect reinforcers (Table 3; Figure 5b) also decreased across weeks of training $(p<0.001)$ but was unaffected by diet $(p=0.627)$.

\section{Post-Injury RGT Choice}

A

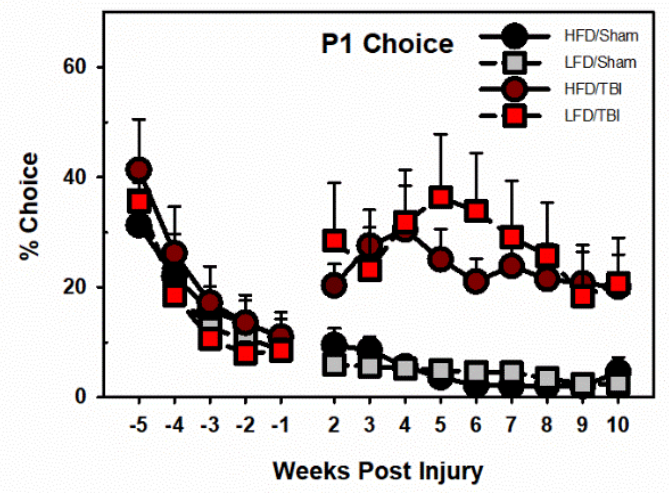

C

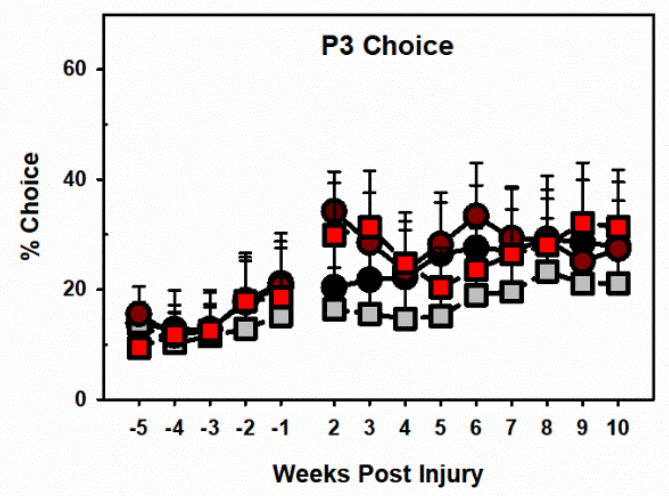

B

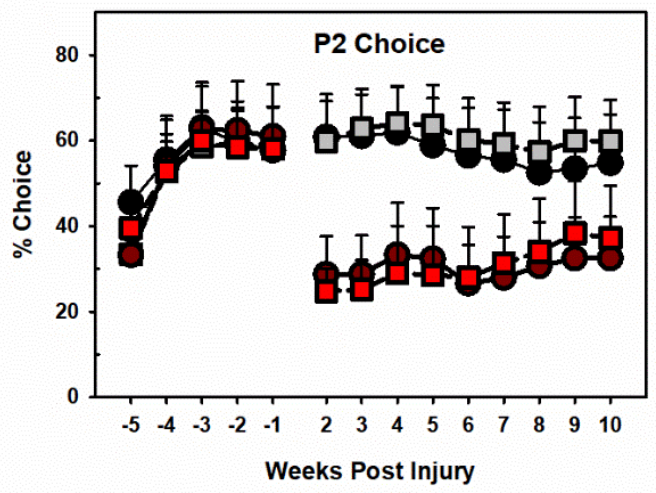

D

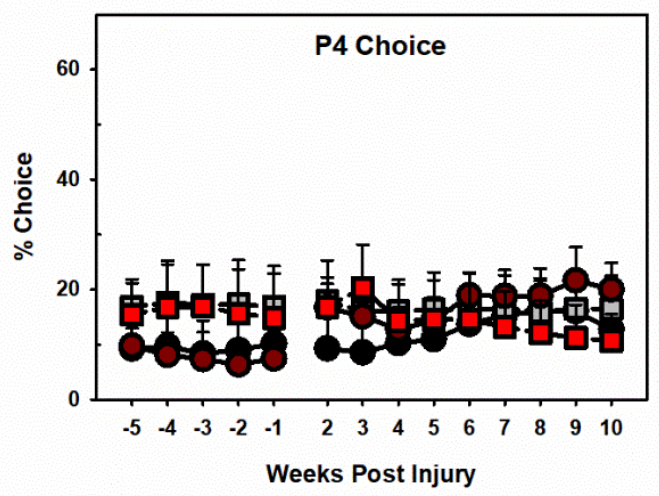

Figure 3. The effects of diet and injury on choice of the P1 (A), P2 (B), P3 (C), and P4 (D) options on the Rodent Gambling Task (RGT). TBI increased suboptimal choice $(p<0.001)$ and risky choice $(p=0.017)$ and decreased optimal choice $(p<0.001)$. The HFD caused slow, progressive deficits on the $\mathrm{P} 2(p<0.001)$ and $\mathrm{P} 4$ options $(p=0.003)$ but not the $\mathrm{P} 1(p=0.268)$ or P3 options $(p=0.111)$.

P1 Choice. TBI increased choice of the suboptimal P1 option (Table 4; Figure 3a) regardless of diet $(p<0.001)$. Choice of the P1 option also decreased across weeks $(p<0.001)$; this decrease was more pronounced for the HFD rats $(p=0.014)$. TBI did not interact with the HFD to produce stronger deficits $(p=0.268)$. 
P2 Choice. Both TBI $(p<0.001)$ and HFD $(p<0.001)$ decreased choice of the optimal P2 option (Table 5; Figure 3b). Although the TBI rats experienced slight recovery over time $(p=$ 0.004), the HFD produced larger decreases in P2 choice as weeks progressed $(p<0.001)$. TBI and the HFD interacted to slightly attenuate deficits compared to TBI and LFD $(p<0.001)$.

P3 Choice. Both TBI $(p=0.017)$ and HFD $(p=0.017)$ increased choice of the risky P3 option (Table 6; Figure 3c). Generally, choice of the P3 option increased across weeks ( $p=$ 0.001) but did not increase for the HFD/TBI group specifically $(p=0.111)$.

P4 Choice. The HFD $(p<0.001)$ increased choice of the risky P4 option (Table 7; Figure 3d) and further increased across weeks $(p<0.001)$. Specifically, the HFD/TBI group experienced the most pronounced increase in P4 choice across weeks $(p=0.003)$, but TBI alone did not increase choice $(p=0.548)$. The TBI alone also caused a decrease in P4 choice across weeks $(p<0.001)$. 

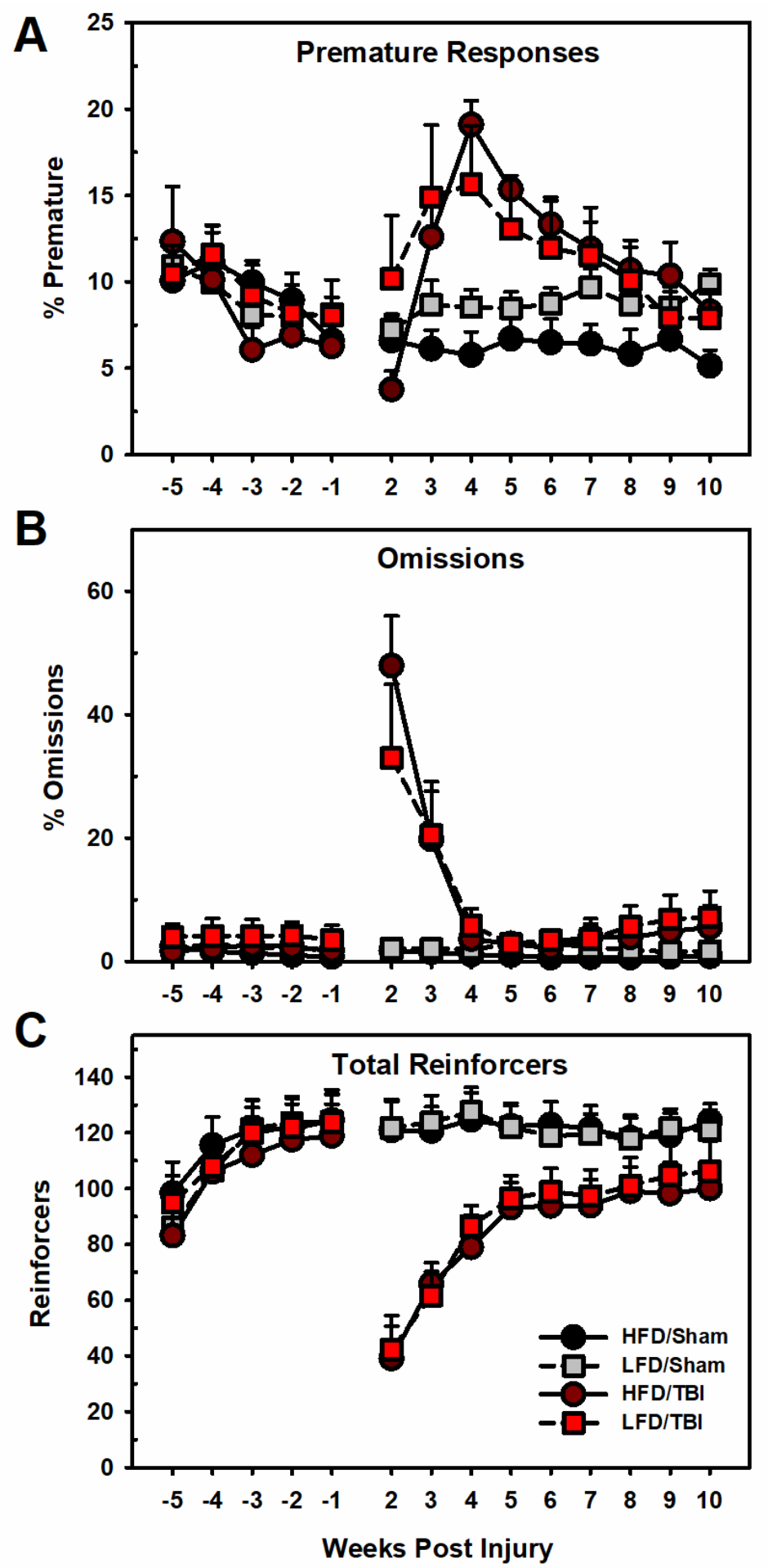

Figure 4. The effects of diet and injury on premature responses (A), omissions (B), and total reinforcers earned $(C)$ on the Rodent Gambling Task. The TBI/HFD rats had the most pronounced impairments on prematures $(p=0.027)$ and omissions $(p=0.005)$. However, only injury (but not diet) impaired overall reinforcement during the task $(p<0.001)$. 


\section{Post-Injury RGT Premature Responses, Omissions, and Reinforcers}

Premature Responses. TBI caused an initial decrease in premature responses that resulted in a net decrease in premature responses (Table 8; Figure 4a) as a result of injury compared to the sham group $(p=0.010)$. However, the TBI/HFD increased in prematures across weeks $(p=0.027)$.

Omissions. TBI rats decreased in omissions (Table 9; Figure 4b) across weeks ( $p<$ $0.001)$ after an initial increase, and this effect was most pronounced for the HFD/TBI group ( $p=$ $0.005)$.

Reinforcers. TBI rats decreased in reinforcers earned (Table 10; Figure 4c) on the task ( $p$ $<0.001)$ regardless of diet $(p=0.656)$ but experienced some recovery over time $(p<0.001)$.

\section{Post-Injury RGT Psychomotor Variables}

A

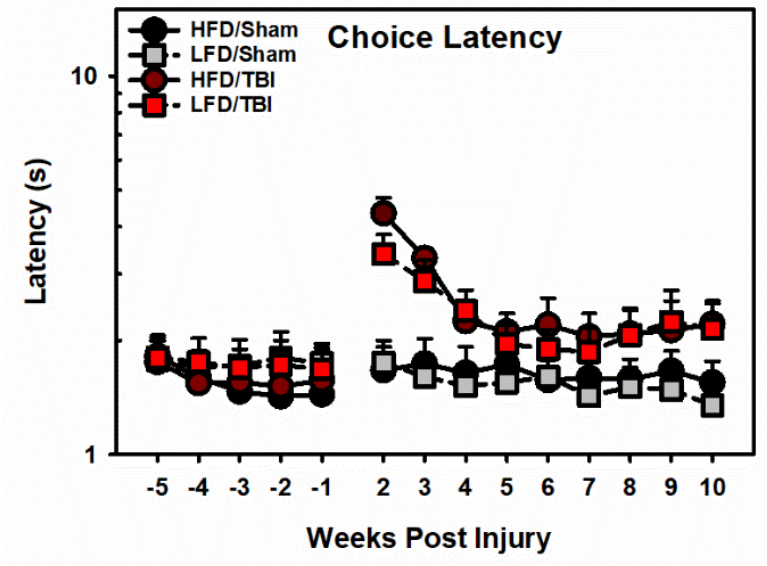

B

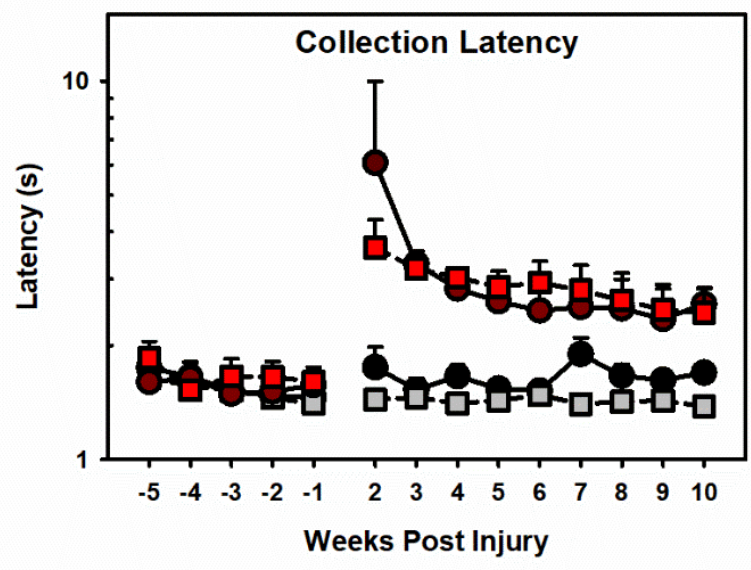

Figure 5. The effects of diet and injury on latency to choose a response (A) and collect reinforcers (B) on the Rodent Gambling Task. Data presented are graphed on a log scale. TBI caused transient increases in both choice latency $(p=0.008)$ and collection latency $(p=0.003)$. Only collection latency deficits were further exacerbated by the HFD $(p<0.001)$. 
Choice latency. Choice latency (Table 11; Figure 5a) was transiently increased by TBI ( $p$ $=0.008)$ but generally decreased across weeks $(p<0.001)$. This decrease was most pronounced for the HFD/TBI rats $(p<0.001)$.

Collection latency. Similarly, collection latency (Table 12; Figure 5b) was initially increased by TBI $(p=0.003)$ but partially recovered across weeks $(p<0.001)$ most notably for HFD/TBI rats $(p<0.001)$.

\section{Immunohistochemistry}

Inter-rater reliability for cell counts was established using a second rater for each area of interest. Although there was a statistical difference between the independent ratings, $t(159)=$ $4.45, p<0.001$, such that the second rater counted 2.31 fewer microglia cells on average, the two ratings were highly correlated, $r=0.91, p<0.001$.

Orbitofrontal cortex. Both TBI $(F(1,132)=80.98, p<0.001)$ and the $\operatorname{HFD}(F(1,132)=$ $5.87, p=0.029)$ significantly increased microglia count in the OFC but did not interact $(F(1$, $132)=0.002, p=0.967)$ to worsen deficits (Figure 6a).

Perilesion area. Both TBI $(F(1,137)=22.26, p<0.001)$ and the $\operatorname{HFD}(F(1,137)=$ 25.43, $p<0.001)$ significantly increased microglia count in the perilesion area and interacted $(F(1,137)=5.90, p=0.017)$ to produce the strongest increase in conjunction (Figure 6b)

Hippocampus. There was no effect of $\operatorname{TBI}(F(1,67)=2.21, p=0.823)$ or $\operatorname{HFD}(F(1,67)$ $=2.013(p=0.161)$ on microglia count in the hippocampus and no interaction $(F(1,67)=0.04, p$ $=0.847)$ of the two variables (Figure 6c). 

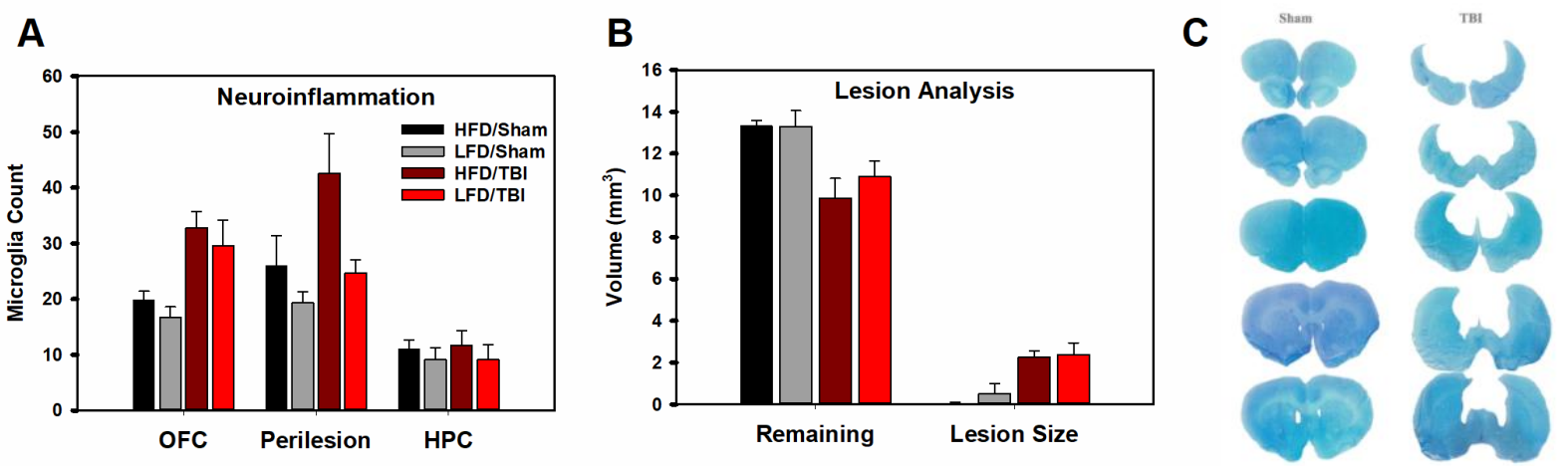

Figure 6. Effects of diet and injury on microglia count in the orbitofrontal cortex (OFC), perilesion area, and hippocampus (HPC; A) and on remaining frontal brain volume and lesion size (B). TBI increased microglia in the OFC $(p<0.001)$ but did not interact with diet $(p=$ $0.967)$. TBI also increased microglia in the perilesion area $(p<0.001)$ and was exacerbated by the HFD $(p=0.017)$. Neither diet $(p=0.161)$ nor injury $(p=0.823)$ affected microglia in the HPC. TBI also decreased remaining frontal brain volume $(p<0.001)$ and increased lesion size $(p$ $<0.001)$ but did not interact with diet $(p=0.57, p=0.693$. A sample sham and TBI brain are also depicted $(\mathrm{C})$, showing the 5 positions that were stained with thionin for lesion analysis.

\section{Lesion Analysis}

TBI significantly decreased brain volume $(F(1,32)=17.24, p<0.001)$. However, the HFD had no effect on volume $(F(1,32)=0.40, p=0.537)$ and did not interact with TBI $(F(1$, $32)=2.55, p=0.454$ ) to worsen tissue loss (Figure 6d). Similarly, TBI increased volume of tissue loss $(F(1,32)=27.12, p<0.001)$, but HFD had no effect on tissue loss $(F(1,32)=0.65, p=$ 0.426, and did not interact with $\operatorname{TBI}(F(1,32)=0.16, p=0.693$. Inter-rater reliability for lesion analysis was established using a second rater for each area of interest. The independent ratings were highly correlated, $r=0.97, p<0.001$, and there was no significant difference between the two ratings, $t(49)=1.50, p=0.14$.

\section{Body Weight}

Body weight (Table 13; Figure 7) steadily increased over time for both diet conditions ( $p$ $<0.001)$. However, TBI interacted with week to slow the progression of weight gain after 
injuries $(p<0.001)$ and did not interact with diet $(p=0.85)$. There was no overall effect of diet on body weight $(p=0.21)$.

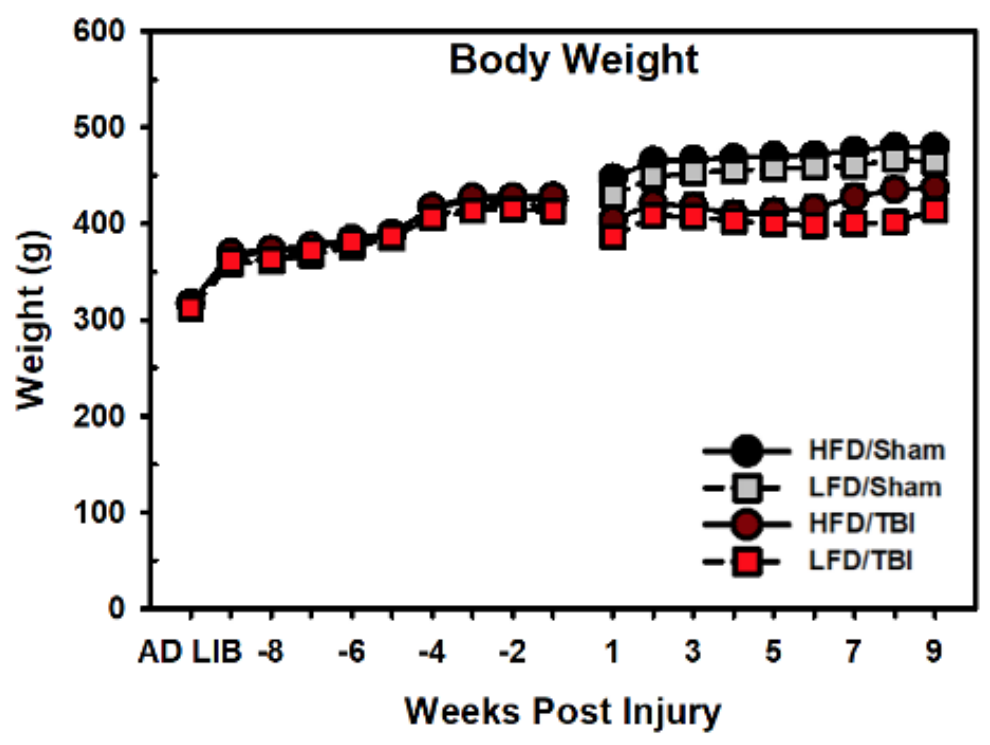

Figure 7. Effects of diet and injury on body weight. TBI decreased the progression of weight gain over time after injuries $(p<0.001)$ but did not interact with the HFD $(p=0.85)$. 


\section{DISCUSSION}

The purpose of this experiment was to determine whether HFD consumption alters behavior and brain function and how those changes might interact with brain injury. We hypothesized that diet-induced neuroinflammation would drive an interactive effect of HFD and TBI. At the level of the brain, the HFD and TBI in combination most robustly increased microglia expression (Figure 6a). However, this increase in inflammation was not reflected by functional consequences on our operant gambling task. We found large-magnitude increases in risky decision-making and motor impulsivity after TBI, but there were no clear detrimental effects of the HFD on behavior. The dietary manipulation did cause some minor changes in behavior that progressed slowly (e.g., decrease in optimal choice over time), but the number of reinforcers earned during the operant task did not differ based on diet (Figure 4c). These collective data indicate that although the HFD did cause subtle changes in decision-making, diet did not produce meaningful impairments that translated into poor task performance and may not have major implications for TBI populations. We also found that HFD had no effect on lesion size in the prefrontal cortex (Figure 6b).

Overall, the HFD worsened TBI-induced inflammation without functional consequence. Although inflammation has been proposed as a mechanism that drives chronic deficits that occur after TBI (Loane, Kumar, Stoica, Cabatbat, \& Faden, 2014; Vonder Haar et al., 2016), our data suggest a disconnect between inflammation and behavior. If risky decision-making and motor impulsivity were driven by inflammation, then differences in microglia expression between dietary conditions should have been reflected in task performance. The regions of interest where we saw an increase in microglia count strengthen this conclusion. First, the null effects of injury on hippocampal microglia confirm that the inflammatory response to injury was focal. In the 
OFC (Zeeb \& Winstanley, 2013), the brain area that regulates optimal decision-making on the RGT, injury and diet both increased inflammation but did not interact. Thus, it logically follows that injury and diet did not interact to worsen optimal decision-making on the RGT. A plausible alternative explanation is that deficits on the RGT were primarily driven by tissue loss in the prefrontal cortex, particularly given that diet and injury interacted to produce the most robust microglia response only in the perilesion area. It is also possible that the null effects of the HFD on behavior are a product of the methodology of the current study. Methodological differences from past literature include task-specific subtleties, types of chronic dysfunction, injury severity, inflammatory manipulation, and calorie restriction.

\section{Methodological Differences}

Task-specific effects. Because diet effects on frontally-mediated cognitive function have been reported in experiments from other labs, it is important to consider task-specific differences between similar work. HFD exposure doubled premature responses compared to a control diet using the 5-CSRTT, an operant task that measures accuracy, attention, and impulsivity (Adams et al., 2015). Notably, the task design allowed for a more powerful detection of motor impulsivity than the RGT. During the 5-CSRTT, rats must respond extremely quickly to a 0.5-s illumination of one of the holes in the chamber (Carli et al., 1983). Responses made during the 5s intertrial interval are considered premature. Thus, the need to withhold responses is pitted against a competing need to respond quickly to receive reinforcement. The RGT has a much longer stimulus duration (10 s), and therefore does not capture impulsive responding as powerfully. Further, the overall accuracy on the 5-CSRTT (i.e., nosepoking correctly in the illuminated hole) was unaffected by the HFD. This is consistent with our finding that diet exposure did not affect overall task performance on the RGT. The effect of diet on reinforcers 
earned was not included in a previous report, but HFD similarly had no effect on total trials performed, reinforcer collection latency, or omissions (Adams et al., 2015). This suggests that the HFD affects task-specific impulsivity rather than overall motivation, attention, and task engagement.

Detrimental effects of HFD consumption were also observed using operant tasks that measure impulsive choice, such as delay discounting (Steele et al., 2017). Delay discounting, or the subjective decrease in the value of a reinforcer as the delay to receiving the reinforcer increases, is studied in laboratory settings with both humans and rodents by providing a choice between a small, immediate reinforcer and a larger, delayed reinforcer (Frost \& McNaughton, 2017; Rachlin, Raineri, \& Cross, 1991). Choice impulsivity is often defined as steeper decreases in subjective value as the delay preceding a reinforcer increases (Rung \& Madden, 2018). Interestingly, HFD consumption reduced overall reinforcers earned on the delay discounting task, indicating that the diet caused meaningful impairments (Steele, Pirkle, Davis, \& Kirkpatrick, 2019). Greater delay discounting was also reported in humans (Dixon et al., 2005) and rats with a TBI (Vonder Haar et al., 2017). Most notably, rats with mild TBIs did not show chronic deficits in the Morris Water Maze, a measure of hippocampal-dependent spatial memory, but still displayed chronic impulsive choice on the delay discounting task (Vonder Haar et al., 2017). Therefore, HFD and TBI may be more likely to produce compounding deficits on a more sensitive measure of psychiatric-like impairment, such as delay discounting. Although motor impulsivity, choice impulsivity, and risky decision-making are all related constructs, subtle differences in operant tasks and feeding regimens may drive the different effects of HFD seen between the current study and prior research. Further, in some experiments, HFD-induced impairments in operant responding may be confounded by weight gain (Burokas et al., 2018; 
Sharma, Fernandes, \& Fulton, 2013). In the current study, body weight was controlled using calorie matching and did not differ between diet conditions (Figure 7). In addition to these more subtle differences in task design, different types of chronic dysfunction may be more targeted by the interaction of TBI and diet.

Type of chronic dysfunction. In the current study, we focused on only two behaviors: risky decision-making and motor impulsivity. Neither of these broad, frontally-mediated behaviors were worsened by HFD consumption after brain injury. However, other studies have found interactions between TBI and diet using other types of behaviors and physiological measures that model different types of chronic dysfunction. For example, free access to an HFD in conjunction with TBI worsened insulin resistance in rodents (Karelina, Sarac, Freeman, Gaier, \& Weil, 2016) as well as hippocampal-dependent task performance (Hoane et al., 2011). It is possible that diet could affect other chronic conditions after TBI, such as epilepsy, because it worsens physiological symptoms including oxidative stress (Alzoubi et al., 2018). A fuller examination of other chronic deficits, such as epilepsy, neurodegeneration, hippocampaldependent function, metabolism, and motor function may be necessary to more fully understand how HFDs can interact with brain injury to worsen deficits.

Injury severity. Another methodological difference that may have driven the null effects of HFD on behavior in the current study is injury severity. In a previous study where HFD consumption exacerbated injury (Hoane et al., 2011), milder CCI injuries were used (2 mm compression of the cortex at $2.75 \mathrm{~m} / \mathrm{s}$ ) compared to our protocols $(2.5 \mathrm{~mm}$ compression at 3 $\mathrm{m} / \mathrm{s}$ ). HFD consumption has also exacerbated deficits resulting from mild concussions using a weight drop method (Mychasiuk, Hehar, Ma, et al., 2015). Although it is possible that the relatively higher severity of injury in the current study created a ceiling effect that obscured any 
potential detrimental effects of the diet, we were able to exacerbate the effects of a severe injury on the RGT using the drug methylphenidate (Vonder Haar et al., in prep). Thus, we may have needed an inflammatory manipulation more potent than diet to see exacerbation of already severe brain injuries.

Inflammatory manipulation. In the current study, diet was used to manipulate inflammation in order to closely resemble risk factors that humans with TBI could encounter in daily life. On the RGT, the HFD did produce low-magnitude decreases in optimal decisionmaking that developed slowly over time (Figure 3b), indicating progressive behavioral impairments. However, a stronger inflammatory manipulation, such as lipopolysaccharide (LPS) injection/infusion, could be required to amplify and interact these deficits with brain injury. LPS is extracted from the cell wall of Gram-negative bacteria (e.g., e. coli) and produces symptoms that mimic infection, when delivered exogenously (Doe \& Henson, 1978; Doe, Yang, Morrison, Betz, \& Henson, 1978). Importantly, LPS administration activates microglia, which mediate the release of pro-inflammatory signaling molecules (Hetier et al., 1988). Peripheral LPS administration 20 days after TBI can exacerbate memory deficits, depressive symptoms, and microglia expression in mice (Fenn et al., 2014; Muccigrosso et al., 2016). Compared to the HFD effects in the current study, LPS also produces more robust microglia activation in the hippocampus (Muccigrosso et al., 2016).

However, HFD consumption in the current study increased microglia counts at a similar or greater magnitude compared to LPS in the prefrontal cortex (Smith, Schmeltzer, Packard, Sah, \& Herman, 2016; Weng, Dong, Wang, Yi, \& Geng, 2019), the brain area heavily involved in our behavioral tasks that should interact with injury. If inflammation in key frontal brain areas (i.e., OFC, prefrontal cortex) drives deficits seen after TBI, then inflammation alone should produce 
similar behavior deficits. However, unpublished data from our lab show that chronic LPS injection alone increased frontal microglia expression but did not impair motivation on a progressive ratio operant task. Further, post-injury treatment with minocycline, an antiinflammatory drug that inhibits microglia (Tikka, Fiebich, Goldsteins, Keinanen, \& Koistinaho, 2001), did not rescue TBI-induced motor impulsivity (Pechacek et al., in prep), although the drug has been shown to attenuate inflammation and alcohol dependence after injury (Karelina et al., 2018). In summary, the HFD produced an inflammatory response consistent with a more direct manipulation of the immune system in the prefrontal cortex without resulting in behavioral deficits of a comparable magnitude. This indicates that inflammation plays a task-specific role in dysfunction after brain injury. Some behaviors, such as spatial memory (Brothers et al., 2013) and depressive behaviors (Weng et al., 2019), are affected by inflammatory manipulations, but we found that risky decision-making and motor impulsivity were not. These behaviors and associated brain regions are fairly under studied in the assessment of both TBI and diet. The HPC tends to be more sensitive to detrimental manipulations because it is one of the most vulnerable and plastic regions of the brain (Anand \& Dhikav, 2012); hippocampal-dependent behaviors, such as the Morris Water Maze, are commonly used to assess function after preclinical TBI. Although it is possible that a stronger inflammatory manipulation, such as LPS, is necessary to exacerbate deficits specific to frontal brain regions, it is more likely that chronic inflammation simply does not worsen some of these frontally-mediated behavioral outcomes after TBI.

Calorie restriction. Alternatively, whereas the present study used HFD in the context of food restriction, HFD manipulations can be strengthened using ad-libitum feeding, which is more common in experiments that use non-operant tasks. In a previous experiment where HFD 
exacerbated behavioral deficits and lesion size after CCI injury in rats, a spatial navigation task was used, which allowed for the rats to have free access to food (Hoane et al., 2011). Free access to HFD also induced spatial and working memory deficits (Greenwood \& Winocur, 1990; Kanoski \& Davidson, 2010), anxiety-like behavior (Karelina et al., 2016), and neuroinflammation (Jeon et al., 2012; Ledreux et al., 2016). In contrast, the current study used rats food restricted to approximately $85 \%$ of free feeding weight, in order to facilitate motivation for operant tasks. It is possible the high and low fat TBI groups recovered at similar rates because their respective diets were calorie restricted and carefully matched. Indeed, there is evidence to suggest that HFD exposure itself may not be detrimental; rather consumption of large quantities of fatty food drives deficits. For example, free access to HFD in rats has more detrimental effects on body weight, insulin resistance, and glucose tolerance compared to scheduled high-fat meals throughout the day (Bake, Morgan, \& Mercer, 2014). Further, calorie restriction has neuroprotective effects after brain injury (Liu et al., 2017; Loncarevic-Vasiljkovic et al., 2012; Rich et al., 2010) and after LPS administration (Radler, Hale, \& Kent, 2014). Thus, unrestricted access to HFD may be necessary to produce certain behavioral deficits and more closely replicate unhealthy eating patterns in clinical populations.

These findings have optimistic implications for therapeutics that make use of high fat foods, such as the ketogenic diet. The ketogenic diet has recently spiked in popularity and has been tested as a treatment for brain injury in both clinical and preclinical populations (McDougall, Bayley, \& Munce, 2018). In fact, a ketogenic diet was recently was used to alleviate some anxiety and depressive-like behaviors in rats after mild TBI (Salberg, Weerwardhena, Collins, Reimer, \& Mychasiuk, 2019). Although the current study did not provide evidence for dietary therapeutics, it does suggest that high fat content alone does not 
worsen outcomes after TBI. It is important to note that the ketogenic diet certainly differs from a rodent HFD, particularly when considering carbohydrate composition, but they do have comparable kilocalories from fat (>60\% and $60 \%$ respectively; Urbain et al., 2017). Further, a rodent HFD contains all nutrients essential for survival, whereas high fat intake in humans without proper supervision can cause nutrient deficiencies (Klevay, 2011; Vormann, 2003). Thus, this particular rodent HFD may share more similarities with calorie-restricted consumption of high-fat food (e.g., ketogenic diet) rather than overconsumption of fatty food.

\section{Limitations and Future Directions}

One way to assess the role of food restriction while still using a clinically-relevant method is to provide rats with free access to their respective diets. If rats maintained on free access to HFD for the same time frame after TBI had higher magnitude increases in microglia, this would suggest that our food restriction paradigm was protective against HFD-induced impairments. The effects of free access to HFD on behavioral outcomes would need to be tested using non-operant behavioral measures such as spatial learning mazes (Kanoski \& Davidson, 2010). Alternatively, different vehicles of fat content delivery may also affect results. For example, vegetable fat was used to induce deficits on the delay discounting task (Steele et al., 2017), although this method is more difficult to match to a control diet.

Another limitation in the current study was the quantification of microglia. The IBA-1 stain was used to quantify microglia regardless of phenotype (Ito et al., 1998). However, microglia exist in multiple phenotypes and have anti-inflammatory states in addition to proinflammatory states (Tang \& Le, 2016). A CD-68 stain only tags active, proinflammatory microglia (Hopperton, Mohammad, Trépanier, Giuliano, \& Bazinet, 2018) and could thus be used to determine if the diet manipulation actually induced a pro-inflammatory state. One final 
future direction is correlating microglia counts with individual behavior. Identifying underlying causes of individual differences in response to TBI was a concern of this experiment. Although the present results do not indicate that differences in fat content drive individual differences in functional outcomes, it is possible that each rat differed in their inflammatory response to both TBI and diet. A correlation between microglia and individual behavior on the RGT would identify a potential role of inflammation in driving individual differences in functional outcome after injury.

In human populations, another contributor to individual differences in recovery from TBI is demographic variables. We were interested in studying diet in conjunction with TBI because low SES, which is often accompanied by poor nutritional quality, is associated with poorer outcomes after injury. However, SES is a broad construct that encompasses a variety of other factors that may worsen recovery, including access to resources and medical care. In the current study, we were unable to find evidence that fat content of food alone exacerbates TBI-induced behavioral deficits in a meaningful way. Another demographic variable that plays an important role in recovery after TBI in clinical populations is sex (Dwyer \& Katz, 2018; King, 2014). This work was piloted in male rates because there are sex differences in patterns of recovery and inflammation after CCI injuries (Taylor, Tio, Paydar, \& Sutton, 2018). When the lab is reliably capable of exacerbating TBI-induced deficits using inflammatory manipulations in males, these techniques can more readily be applied to female rats. However, because we did not find compelling evidence to continue studying the interactive effects of diet and TBI, there is little benefit to extending these particular techniques to females, given that male rats (Lindqvist et al., 2006) and mice (Hwang et al., 2010) are more sensitive to the detrimental effects of HFDs than females. Other avenues of TBI research that might benefit more from female replication include 
the use of transcranial direct current stimulation (Martens et al., 2019) and environmental manipulations (Modrak et al., in prep; O’Hearn et al., in prep) to successfully treat deficits in male rats. Given the bias against female animals in neuroscience research that has dominated previous decades (Zucker \& Beery, 2010), it is important for females to be represented in TBI research moving forward.

\section{Conclusions}

Although some methodological subtleties, such as the task design, injury severity, and food restriction, may have reduced the power of the study, we are confident in these null findings because we successfully replicated our model of TBI-induced deficits. We see consistent deficits in optimal decision-making and impulsivity using the RGT after TBI; manipulations that worsen post-injury recovery should exacerbate these deficits. Overall, these results suggest that an HFD may not be functionally detrimental in conjunction with brain injury when assessing specific psychiatric-like behaviors. Given that the fat content does increase inflammation, this raises questions about the role of inflammation in TBI-induced deficits. It is possible that inflammation does not drive certain deficits after brain injury but simply occurs alongside these deficits. Because inflammation is seen in the human brain for years after injury (Gentleman et al., 2004; Johnson et al., 2013; Ramlackhansingh et al., 2011), this question is important to resolve to effectively characterize and treat the psychiatric-like symptoms that result from TBI. 
APPENDIX A: Coefficient Tables

TABLE 1: P1-4 Choice Coefficients (Pre-Injury)

\begin{tabular}{|c|c|c|c|}
\hline Variable & $\beta$ & $t$ & $p$ \\
\hline \multicolumn{4}{|l|}{ P1 Choice } \\
\hline Diet & 0.08 & 1.48 & 0.137 \\
\hline Week & -0.34 & -9.50 & $<0.001 * * *$ \\
\hline Diet x Week & 0.03 & 0.57 & 0.566 \\
\hline \multicolumn{4}{|l|}{ P2 Choice } \\
\hline Diet & 0.10 & 1.87 & 0.062 \\
\hline Week & 0.22 & 6.21 & $<0.001 * * *$ \\
\hline Diet x Week & -0.01 & -0.27 & 0.786 \\
\hline \multicolumn{4}{|l|}{ P3 Choice } \\
\hline Diet & 0.06 & 1.23 & 0.219 \\
\hline Week & 0.03 & 0.71 & 0.477 \\
\hline Diet x Week & 0.02 & 0.40 & 0.069 \\
\hline \multicolumn{4}{|l|}{ P4 Choice } \\
\hline Diet & -0.32 & -6.41 & $<0.001 * * *$ \\
\hline Week & -0.05 & -1.29 & 0.197 \\
\hline Diet x Week & -0.01 & -0.21 & 0.36 \\
\hline
\end{tabular}

Note: $*$ denotes $p<0.05$, ** denotes $p<0.01$, *** denotes $p<0.001$ 
TABLE 2: Prematures, Omissions, and Reinforcers (Pre-Injury)

\begin{tabular}{|c|c|c|c|}
\hline Variable & $\beta$ & $t$ & $p$ \\
\hline \multicolumn{4}{|l|}{ Prematures } \\
\hline Diet & -0.20 & -0.80 & 0.429 \\
\hline Week & -0.17 & -5.25 & $<0.001 * * *$ \\
\hline Diet x Week & -0.003 & -0.07 & 0.946 \\
\hline \multicolumn{4}{|l|}{ Omissions } \\
\hline Diet & -0.34 & -1.52 & 0.139 \\
\hline Week & -0.05 & -1.32 & 0.187 \\
\hline Diet x Week & -0.10 & -2.02 & $0.044 *$ \\
\hline \multicolumn{4}{|l|}{ Reinforcers } \\
\hline Diet & -0.02 & -0.07 & 0.943 \\
\hline Week & 0.34 & 11.96 & $<0.001 * * *$ \\
\hline Diet x Week & -0.06 & -1.42 & 0.155 \\
\hline
\end{tabular}


TABLE 3: Psychomotor Variables (Pre-injury)

\begin{tabular}{lccc}
\hline Variable & $\beta$ & $t$ & $p$ \\
\hline Choice Latency & & & 0.502 \\
Diet & -0.10 & -0.68 & $0.027^{*}$ \\
Week & -0.01 & -2.21 & $<0.001^{* * *}$ \\
Diet x Week & -0.04 & -5.51 & \\
Collection Latency & & & 0.627 \\
Diet & -0.06 & -0.49 & $<0.001 * * *$ \\
Week & -0.02 & -3.78 & 0.198 \\
Diet x Week & 0.01 & 1.29 & \\
\hline
\end{tabular}


TABLE 4: P1 Choice Coefficients (Post-Injury)

\begin{tabular}{lccc}
\hline Variable & $\beta$ & $t$ & $p$ \\
\hline Diet & 0.014 & 0.47 & 0.642 \\
Injury & 0.28 & 8.93 & $<0.001 * * *$ \\
Week & -0.07 & -3.73 & $<0.001 * * *$ \\
Baseline & 0.93 & 134.57 & $<0.001^{* * *}$ \\
Diet x Injury & -0.049 & -1.11 & 0.268 \\
Diet x Week & -0.07 & -2.45 & $0.014 *$ \\
Injury x Week & -0.019 & -0.61 & 0.542 \\
Diet x Injury x Week & 0.064 & 1.49 & 0.138 \\
\hline
\end{tabular}

TABLE 5: P2 Choice Coefficients (Post-Injury)

\begin{tabular}{lccc}
\hline Variable & $\beta$ & $t$ & $p$ \\
\hline Diet & -0.16 & -5.40 & $<0.001^{* * * *}$ \\
Injury & -0.44 & -13.88 & $<0.001 * * *$ \\
Week & -0.17 & -0.88 & 0.379 \\
Baseline & 0.93 & 134.57 & $<0.001^{* * *}$ \\
Diet x Injury & 0.25 & 5.64 & $<0.001^{* * *}$ \\
Diet x Week & -0.092 & -3.30 & $<0.001 * * *$ \\
Injury x Week & 0.09 & 2.89 & $0.004 * *$ \\
Diet x Injury x Week & -0.001 & -0.03 & 0.977 \\
\hline
\end{tabular}


TABLE 6: P3 Choice Coefficients (Post-Injury)

\begin{tabular}{lccc}
\hline Variable & $\beta$ & $t$ & $p$ \\
\hline Diet & 0.07 & 2.39 & $0.017^{*}$ \\
Injury & 0.008 & 2.40 & $0.017^{*}$ \\
Week & 0.07 & 3.42 & $0.001^{* *}$ \\
Baseline & 0.93 & 134.57 & $<0.001^{* * *}$ \\
Diet x Injury & -0.07 & -1.59 & 0.111 \\
Diet x Week & 0.05 & 1.76 & 0.079 \\
Injury x Week & -0.03 & -1.07 & 0.284 \\
Diet x Injury x Week & -0.11 & -2.46 & $0.014^{*}$ \\
\hline
\end{tabular}

TABLE 7: P4 Choice Coefficients (Post-Injury)

\begin{tabular}{lccc}
\hline Variable & $\beta$ & $t$ & $p$ \\
\hline Diet & 0.10 & 3.38 & $<0.001^{* * * *}$ \\
Injury & 0.02 & 0.60 & 0.548 \\
Week & -0.01 & -0.40 & $<0.001^{* * *}$ \\
Baseline & 0.93 & 134.57 & 0.472 \\
Diet x Injury & 0.032 & 0.73 & $<0.001^{* * *}$ \\
Diet x Week & 0.10 & 3.60 & $<0.001^{* * * *}$ \\
Injury x Week & -0.12 & -3.77 & $0.003 * *$ \\
Diet x Injury x Week & 0.13 & 2.94 & \\
\hline
\end{tabular}


TABLE 8: Premature Response Coefficients (Post-Injury)

\begin{tabular}{lccc}
\hline Variable & $\beta$ & $t$ & $p$ \\
\hline Diet & -0.23 & -2.73 & $0.010^{*}$ \\
Injury & -0.21 & -2.40 & $0.023^{*}$ \\
Week & 0.08 & 1.98 & $0.048^{*}$ \\
Baseline & 0.53 & 15.84 & $<0.001^{* * *}$ \\
Diet x Injury & 0.36 & 2.84 & $0.008^{* *}$ \\
Diet x Week & -0.06 & -1.14 & 0.253 \\
Injury x Week & -0.01 & -0.24 & 0.811 \\
Diet x Injury x Week & 0.19 & 2.21 & $0.027 *$ \\
\hline
\end{tabular}

TABLE 9: Omission Coefficients (Post-Injury)

\begin{tabular}{lccc}
\hline Variable & $\beta$ & $t$ & $p$ \\
\hline Diet & 0.07 & -0.34 & 0.735 \\
Injury & -0.16 & -0.70 & 0.487 \\
Week & -0.08 & -2.58 & $0.010^{*}$ \\
Baseline & 0.61 & 5.89 & $<0.001^{* * *}$ \\
Diet x Injury & -0.07 & -0.25 & 0.807 \\
Diet x Week & 0.001 & 0.03 & 0.980 \\
Injury x Week & -0.28 & -5.43 & $<0.001^{* * *}$ \\
Diet x Injury x Week & -0.20 & -2.82 & $0.005^{* *}$ \\
\hline
\end{tabular}


TABLE 10: Reinforcer Coefficients (Post-Injury)

\begin{tabular}{lccc}
\hline Variable & $\beta$ & $t$ & $p$ \\
\hline Diet & -0.03 & -0.27 & 0.792 \\
Injury & -0.44 & -3.65 & $0.001^{* *}$ \\
Week & -0.04 & -1.35 & 0.177 \\
Baseline & 0.59 & 12.75 & $<0.001^{* * *}$ \\
Diet x Injury & 0.07 & 0.50 & 0.656 \\
Diet x Week & 0.03 & 0.65 & 0.514 \\
Injury x Week & 0.53 & 12.20 & $<0.001 * * *$ \\
Diet x Injury x Week & -0.07 & -1.08 & 0.280 \\
\hline
\end{tabular}

TABLE 11: Choice Latency (Post-Injury)

\begin{tabular}{lccc}
\hline Variable & $\beta$ & $t$ & $p$ \\
\hline Diet & 0.12 & 1.87 & 0.071 \\
Injury & 0.20 & 2.84 & $0.008^{* *}$ \\
Week & -0.06 & -10.94 & $<0.001^{* * *}$ \\
Baseline & 0.34 & 12.56 & $<0.001$ \\
Diet x Injury & -0.04 & -0.41 & 0.684 \\
Diet x Week & 0.003 & 0.42 & 0.675 \\
Injury x Week & -0.01 & -1.45 & 0.148 \\
Diet x Injury x Week & -0.06 & -4.50 & $<0.001^{* * *}$ \\
\hline
\end{tabular}


TABLE 12: Collection Latency (Post-Injury)

\begin{tabular}{lccc}
\hline Variable & $\beta$ & $t$ & $p$ \\
\hline Diet & -0.04 & -0.52 & 0.604 \\
Injury & 0.38 & 3.23 & $0.003^{* *}$ \\
Week & -0.02 & -5.08 & $<0.001^{* * *}$ \\
Baseline & 0.57 & 12.30 & $<0.001^{* * *}$ \\
Diet x Injury & -0.003 & -0.02 & 0.983 \\
Diet x Week & -0.01 & -1.99 & $0.047^{*}$ \\
Injury x Week & -0.10 & -13.24 & $<0.001^{* * *}$ \\
Diet x Injury x Week & -0.11 & -10.80 & $<0.001^{* * *}$ \\
\hline
\end{tabular}

\section{TABLE 13: Body Weight}

\begin{tabular}{lccc}
\hline Variable & $\beta$ & $t$ & $p$ \\
\hline Diet & 0.27 & 1.29 & 0.208 \\
Injury & -0.56 & -2.90 & $0.013^{* *}$ \\
Week & 0.95 & 41.40 & $<0.001^{* * *}$ \\
Diet x Injury & 0.05 & 0.19 & 0.849 \\
Diet x Week & 0.07 & 2.09 & $0.037^{*}$ \\
Injury x Week & -0.57 & -16.42 & $<0.001^{* * *}$ \\
Diet x Injury x Week & 0.07 & 1.40 & 0.163 \\
\hline
\end{tabular}




\section{REFERENCES}

Adams, W. K., Sussman, J. L., Kaur, S., D'Souza A, M., Kieffer, T. J., \& Winstanley, C. A. (2015). Long-term, calorie-restricted intake of a high-fat diet in rats reduces impulse control and ventral striatal D2 receptor signalling - two markers of addiction vulnerability. Eur J Neurosci, 42(12), 3095-3104. doi:10.1111/ejn.13117

Adler, N. E., \& Newman, K. (2002). Socioeconomic disparities in health: pathways and policies. Health Aff (Millwood), 21(2), 60-76. doi:10.1377/hlthaff.21.2.60

Alderman, N. (2003). Contemporary approaches to the management of irritability and aggression following traumatic brain injury. Neuropsychol Rehabil, 13(1-2), 211-240. doi:10.1080/09602010244000327

Alzoubi, K. H., Hasan, Z. A., Khabour, O. F., Mayyas, F. A., Al Yacoub, O. N., Banihani, S. A., ... Alrabadi, N. (2018). The effect of high-fat diet on seizure threshold in rats: Role of oxidative stress. Physiol Behav, 196, 1-7. doi:10.1016/j.physbeh.2018.08.011

Anand, K. S., \& Dhikav, V. (2012). Hippocampus in health and disease: An overview. Ann Indian Acad Neurol, 15(4), 239-246. doi:10.4103/0972-2327.104323

Association, A. P. (2013). Diagnostic and Statistical Manual of Mental Disorders (5th ed.). Washington, DC.

Bake, T., Morgan, D. G., \& Mercer, J. G. (2014). Feeding and metabolic consequences of scheduled consumption of large, binge-type meals of high fat diet in the Sprague-Dawley rat. Physiol Behav, 128(100), 70-79. doi:10.1016/j.physbeh.2014.01.018

Barrus, M. M., Hosking, J. G., Zeeb, F. D., Tremblay, M., \& Winstanley, C. A. (2015). Disadvantageous decision-making on a rodent gambling task is associated with increased motor impulsivity in a population of male rats. J Psychiatry Neurosci, 40(2), 108-117. doi:10.1503/jpn.140045

Bechara, A., Damasio, A. R., Damasio, H., \& Anderson, S. W. (1994). Insensitivity to future consequences following damage to human prefrontal cortex. Cognition, 50(1-3), 7-15. doi:10.1016/0010-0277(94)90018-3

Beilharz, J. E., Maniam, J., \& Morris, M. J. (2015). Diet-Induced Cognitive Deficits: The Role of Fat and Sugar, Potential Mechanisms and Nutritional Interventions. Nutrients, 7(8), 67196738. doi:10.3390/nu7085307

Brothers, H. M., Bardou, I., Hopp, S. C., Kaercher, R. M., Corona, A. W., Fenn, A. M., . . . Wenk, G. L. (2013). Riluzole partially rescues age-associated, but not LPS-induced, loss of glutamate transporters and spatial memory. J Neuroimmune Pharmacol, 8(5), 10981105. doi:10.1007/s11481-013-9476-2

Burokas, A., Martín-García, E., Espinosa-Carrasco, J., Erb, I., McDonald, J., Notredame, C., . . . Maldonado, R. (2018). Extinction and reinstatement of an operant responding maintained by food in different models of obesity. Addict Biol, 23(2), 544-555. doi:10.1111/adb.12597

Carli, M., Robbins, T. W., Evenden, J. L., \& Everitt, B. J. (1983). Effects of lesions to ascending noradrenergic neurones on performance of a 5-choice serial reaction task in rats; implications for theories of dorsal noradrenergic bundle function based on selective attention and arousal. Behav Brain Res, 9(3), 361-380. doi:10.1016/0166-4328(83)901389

Chase, H. W., Fournier, J. C., Bertocci, M. A., Greenberg, T., Aslam, H., Stiffler, R., . . . Phillips, M. L. (2017). A pathway linking reward circuitry, impulsive sensation-seeking 
and risky decision-making in young adults: identifying neural markers for new interventions. Transl Psychiatry, 7(4), e1096. doi:10.1038/tp.2017.60

Chen, J. Q., Zhang, C. C., Lu, H., \& Wang, W. (2014). Assessment of traumatic brain injury degree in animal model. Asian Pac J Trop Med, 7(12), 991-995. doi:10.1016/s19957645(14)60174-7

Chiu, C. C., Liao, Y. E., Yang, L. Y., Wang, J. Y., Tweedie, D., Karnati, H. K., . . Wang, J. Y. (2016). Neuroinflammation in animal models of traumatic brain injury. J Neurosci Methods, 272, 38-49. doi:10.1016/j.jneumeth.2016.06.018

Cole, J. T., Yarnell, A., Kean, W. S., Gold, E., Lewis, B., Ren, M., . . Watson, W. D. (2011). Craniotomy: true sham for traumatic brain injury, or a sham of a sham? J Neurotrauma, 28(3), 359-369. doi:10.1089/neu.2010.1427

Cooper, D. B., Kennedy, J. E., Cullen, M. A., Critchfield, E., Amador, R. R., \& Bowles, A. O. (2011). Association between combat stress and post-concussive symptom reporting in OEF/OIF service members with mild traumatic brain injuries. Brain Inj, 25(1), 1-7. doi:10.3109/02699052.2010.531692

Cordain, L., Eaton, S. B., Sebastian, A., Mann, N., Lindeberg, S., Watkins, B. A., . . BrandMiller, J. (2005). Origins and evolution of the Western diet: health implications for the 21st century. Am J Clin Nutr, 81(2), 341-354. doi:10.1093/ajcn.81.2.341

Darmon, N., \& Drewnowski, A. (2008). Does social class predict diet quality? Am J Clin Nutr, 87(5), 1107-1117. doi:10.1093/ajcn/87.5.1107

Dixon, C. E., Clifton, G. L., Lighthall, J. W., Yaghmai, A. A., \& Hayes, R. L. (1991). A controlled cortical impact model of traumatic brain injury in the rat. J Neurosci Methods, 39(3), 253-262. doi:10.1016/0165-0270(91)90104-8

Dixon, M., Jacobs, E., Sanders, S., Guercio, J., Soldner, J., Parker-Singler, S., . . Dillen, J. (2005). Impulsivity, Self-Control, and Delay Discounting in Persons with Acquired Brain Injury. Behavioral Interventions, 20, 101-120. doi:10.1002/bin.173

Doe, W. F., \& Henson, P. M. (1978). Macrophage stimulation by bacterial lipopolysaccharides. I. Cytolytic effect on tumor target cells. J Exp Med, 148(2), 544-556. doi:10.1084/jem.148.2.544

Doe, W. F., Yang, S. T., Morrison, D. C., Betz, S. J., \& Henson, P. M. (1978). Macrophage stimulation by bacterial lipopolysaccharides. II. Evidence for differentiation signals delivered by lipid A and by a protein rich fraction of lipopolysaccharides. J Exp Med, 148(2), 557-568. doi:10.1084/jem.148.2.557

Donat, C. K., Scott, G., Gentleman, S. M., \& Sastre, M. (2017). Microglial Activation in Traumatic Brain Injury. Frontiers in Aging Neuroscience, 9(208). doi:10.3389/fnagi.2017.00208

Dwyer, B., \& Katz, D. (2018). Postconcussion syndrome. In (pp. 163-178).

Dyer, K. F., Bell, R., McCann, J., \& Rauch, R. (2006). Aggression after traumatic brain injury: analysing socially desirable responses and the nature of aggressive traits. Brain Inj, 20(11), 1163-1173. doi:10.1080/02699050601049312

Faul, M., Xu, L., Wald, M. M., Coronado, V., \& Dellinger, A. M. (2010). Traumatic brain injury in the United States: National estimates of prevalence and incidence, 2002-2006. Injury Prevention, 16, A268-A268. doi:doi:10.1136/ip.2010.029215.951

Fenn, A. M., Gensel, J. C., Huang, Y., Popovich, P. G., Lifshitz, J., \& Godbout, J. P. (2014). Immune activation promotes depression 1 month after diffuse brain injury: a role for primed microglia. Biol Psychiatry, 76(7), 575-584. doi:10.1016/j.biopsych.2013.10.014 
Frost, R., \& McNaughton, N. (2017). The neural basis of delay discounting: A review and preliminary model. Neurosci Biobehav Rev, 79, 48-65. doi:10.1016/j.neubiorev.2017.04.022

Fung, T. T., Rimm, E. B., Spiegelman, D., Rifai, N., Tofler, G. H., Willett, W. C., \& Hu, F. B. (2001). Association between dietary patterns and plasma biomarkers of obesity and cardiovascular disease risk. Am J Clin Nutr, 73(1), 61-67. doi:10.1093/ajcn/73.1.61

Garcia, D., Hungerford, G., \& Bagner, D. (2014). Topical Review: A Review of Negative Behavioral and Cognitive Outcomes Following Traumatic Brain Injury in Early Childhood. Journal of pediatric psychology, 40. doi:10.1093/jpepsy/jsu093

Gasco, V., Prodam, F., Pagano, L., Grottoli, S., Belcastro, S., Marzullo, P., . . A Aimaretti, G. (2012). Hypopituitarism following brain injury: when does it occur and how best to test? Pituitary, 15(1), 20-24. doi:10.1007/s11102-010-0235-6

Gentleman, S. M., Leclercq, P. D., Moyes, L., Graham, D. I., Smith, C., Griffin, W. S., \& Nicoll, J. A. (2004). Long-term intracerebral inflammatory response after traumatic brain injury. Forensic Sci Int, 146(2-3), 97-104. doi:10.1016/j.forsciint.2004.06.027

Giskes, K., Avendano, M., Brug, J., \& Kunst, A. E. (2010). A systematic review of studies on socioeconomic inequalities in dietary intakes associated with weight gain and overweight/obesity conducted among European adults. Obes Rev, 11(6), 413-429. doi:10.1111/j.1467-789X.2009.00658.x

Greenwood, C. E., \& Winocur, G. (1990). Learning and memory impairment in rats fed a high saturated fat diet. Behav Neural Biol, 53(1), 74-87. doi:10.1016/0163-1047(90)90831-p

Griffin, S. A., Lynam, D. R., \& Samuel, D. B. (2018). Dimensional conceptualizations of impulsivity. Personal Disord, 9(4), 333-345. doi:10.1037/per0000253

Guillemot-Legris, O., \& Muccioli, G. G. (2017). Obesity-Induced Neuroinflammation: Beyond the Hypothalamus. Trends Neurosci, 40(4), 237-253. doi:10.1016/j.tins.2017.02.005

Guskiewicz, K. M., McCrea, M., Marshall, S. W., Cantu, R. C., Randolph, C., Barr, W., . . . Kelly, J. P. (2003). Cumulative effects associated with recurrent concussion in collegiate football players: the NCAA Concussion Study. Jama, 290(19), 2549-2555. doi:10.1001/jama.290.19.2549

Hamm, R. J., Pike, B. R., Temple, M. D., O'Dell, D. M., \& Lyeth, B. G. (1995). The effect of postinjury kindled seizures on cognitive performance of traumatically brain-injured rats. Exp Neurol, 136(2), 143-148. doi:10.1006/exnr.1995.1091

Hariri, N., \& Thibault, L. (2010). High-fat diet-induced obesity in animal models. Nutr Res Rev, 23(2), 270-299. doi:10.1017/s0954422410000168

Harry, G. J. (2013). Microglia during development and aging. Pharmacol Ther, 139(3), 313-326. doi:10.1016/j.pharmthera.2013.04.013

Hehar, H., Yeates, K., Kolb, B., Esser, M. J., \& Mychasiuk, R. (2015). Impulsivity and Concussion in Juvenile Rats: Examining Molecular and Structural Aspects of the Frontostriatal Pathway. PLoS One, 10(10), e0139842. doi:10.1371/journal.pone.0139842

Hetier, E., Ayala, J., Denèfle, P., Bousseau, A., Rouget, P., Mallat, M., \& Prochiantz, A. (1988). Brain macrophages synthesize interleukin-1 and interleukin-1 mRNAs in vitro. $J$ Neurosci Res, 21(2-4), 391-397. doi:10.1002/jnr.490210230

Hoane, M. R., Swan, A. A., \& Heck, S. E. (2011). The effects of a high-fat sucrose diet on functional outcome following cortical contusion injury in the rat. Behav Brain Res, 223(1), 119-124. doi:10.1016/j.bbr.2011.04.028 
Hopperton, K. E., Mohammad, D., Trépanier, M. O., Giuliano, V., \& Bazinet, R. P. (2018). Markers of microglia in post-mortem brain samples from patients with Alzheimer's disease: a systematic review. Mol Psychiatry, 23(2), 177-198. doi:10.1038/mp.2017.246

Hwang, L. L., Wang, C. H., Li, T. L., Chang, S. D., Lin, L. C., Chen, C. P., . . Chiou, L. C. (2010). Sex differences in high-fat diet-induced obesity, metabolic alterations and learning, and synaptic plasticity deficits in mice. Obesity (Silver Spring), 18(3), 463-469. doi:10.1038/oby.2009.273

Hyder, A., Wunderlich, C., Puvanachandra, P., Gururaj, G., \& Kobusingye, O. (2007). The impact of traumatic brain injuries: A global perspective. NeuroRehabilitation, 22, 341353. doi:10.3233/NRE-2007-22502

Isung, J., Aeinehband, S., Mobarrez, F., Nordström, P., Runeson, B., Asberg, M., . . Jokinen, J. (2014). High interleukin-6 and impulsivity: determining the role of endophenotypes in attempted suicide. Transl Psychiatry, 4(10), e470. doi:10.1038/tp.2014.113

Ito, D., Imai, Y., Ohsawa, K., Nakajima, K., Fukuuchi, Y., \& Kohsaka, S. (1998). Microgliaspecific localisation of a novel calcium binding protein, Iba1. Brain Res Mol Brain Res, 57(1), 1-9. doi:10.1016/s0169-328x(98)00040-0

Jassam, Y. N., Izzy, S., Whalen, M., McGavern, D. B., \& El Khoury, J. (2017). Neuroimmunology of Traumatic Brain Injury: Time for a Paradigm Shift. Neuron, 95(6), 1246-1265. doi:10.1016/j.neuron.2017.07.010

Jeon, B. T., Jeong, E. A., Shin, H. J., Lee, Y., Lee, D. H., Kim, H. J., . . Roh, G. S. (2012). Resveratrol attenuates obesity-associated peripheral and central inflammation and improves memory deficit in mice fed a high-fat diet. Diabetes, 61(6), 1444-1454. doi:10.2337/db11-1498

Johnson, V. E., Stewart, J. E., Begbie, F. D., Trojanowski, J. Q., Smith, D. H., \& Stewart, W. (2013). Inflammation and white matter degeneration persist for years after a single traumatic brain injury. Brain, 136(Pt 1), 28-42. doi:10.1093/brain/aws322

Kanoski, S. E., \& Davidson, T. L. (2010). Different patterns of memory impairments accompany short- and longer-term maintenance on a high-energy diet. J Exp Psychol Anim Behav Process, 36(2), 313-319. doi:10.1037/a0017228

Karelina, K., Nicholson, S., \& Weil, Z. M. (2018). Minocycline blocks traumatic brain injuryinduced alcohol consumption and nucleus accumbens inflammation in adolescent male mice. Brain Behav Immun, 69, 532-539. doi:10.1016/j.bbi.2018.01.012

Karelina, K., Sarac, B., Freeman, L. M., Gaier, K. R., \& Weil, Z. M. (2016). Traumatic brain injury and obesity induce persistent central insulin resistance. Eur J Neurosci, 43(8), 1034-1043. doi:10.1111/ejn.13194

King, N. (2014). Permanent post concussion symptoms after mild head injury: A systematic review of age and gender factors. NeuroRehabilitation, 34. doi:10.3233/NRE-141072

King, N. S., \& Kirwilliam, S. (2011). Permanent post-concussion symptoms after mild head injury. Brain Inj, 25(5), 462-470. doi:10.3109/02699052.2011.558042

Klevay, L. M. (2011). Is the Western diet adequate in copper? J Trace Elem Med Biol, 25(4), 204-212. doi:10.1016/j.jtemb.2011.08.146

Koponen, S., Taiminen, T., Portin, R., Himanen, L., Isoniemi, H., Heinonen, H., . . Tenovuo, O. (2002). Axis I and II psychiatric disorders after traumatic brain injury: a 30-year followup study. Am J Psychiatry, 159(8), 1315-1321. doi:10.1176/appi.ajp.159.8.1315 
Ledreux, A., Wang, X., Schultzberg, M., Granholm, A. C., \& Freeman, L. R. (2016). Detrimental effects of a high fat/high cholesterol diet on memory and hippocampal markers in aged rats. Behav Brain Res, 312, 294-304. doi:10.1016/j.bbr.2016.06.012

Lee, R. S. C., Hoppenbrouwers, S., \& Franken, I. (2019). A Systematic Meta-Review of Impulsivity and Compulsivity in Addictive Behaviors. Neuropsychol Rev, 29(1), 14-26. doi:10.1007/s11065-019-09402-x

Lighthall, J. W. (1988). Controlled cortical impact: a new experimental brain injury model. $J$ Neurotrauma, 5(1), 1-15. doi:10.1089/neu.1988.5.1

Lindqvist, A., Mohapel, P., Bouter, B., Frielingsdorf, H., Pizzo, D., Brundin, P., \& ErlansonAlbertsson, C. (2006). High-fat diet impairs hippocampal neurogenesis in male rats. Eur J Neurol, 13(12), 1385-1388. doi:10.1111/j.1468-1331.2006.01500.x

Liu, Y., Wang, R., Zhao, Z., Dong, W., Zhang, X., Chen, X., \& Ma, L. (2017). Short-term caloric restriction exerts neuroprotective effects following mild traumatic brain injury by promoting autophagy and inhibiting astrocyte activation. Behavioural brain research, 331, 135-142. doi:10.1016/j.bbr.2017.04.024

Loane, D. J., Kumar, A., Stoica, B. A., Cabatbat, R., \& Faden, A. I. (2014). Progressive neurodegeneration after experimental brain trauma: association with chronic microglial activation. J Neuropathol Exp Neurol, 73(1), 14-29. doi:10.1097/nen.0000000000000021

Loncarevic-Vasiljkovic, N., Pesic, V., Todorovic, S., Popic, J., Smiljanic, K., Milanovic, D., .. . Kanazir, S. (2012). Caloric Restriction Suppresses Microglial Activation and Prevents Neuroapoptosis Following Cortical Injury in Rats. PLoS One, 7(5), e37215. doi:10.1371/journal.pone.0037215

Longhi, L., Watson, D. J., Saatman, K. E., Thompson, H. J., Zhang, C., Fujimoto, S., . . McIntosh, T. K. (2004). Ex vivo gene therapy using targeted engraftment of NGFexpressing human NT2N neurons attenuates cognitive deficits following traumatic brain injury in mice. J Neurotrauma, 21(12), 1723-1736. doi:10.1089/neu.2004.21.1723

Manes, F., Sahakian, B., Clark, L., Rogers, R., Antoun, N., Aitken, M., \& Robbins, T. (2002). Decision-making processes following damage to the prefrontal cortex. Brain, 125(Pt 3), 624-639. doi:10.1093/brain/awf049

Martens, K., Pechacek, K., Modrak, C., Milleson, V., Zhu, B., \& Vonder Haar, C. (2019). Cathodal Transcranial Direct-Current Stimulation Selectively Decreases Impulsivity after Traumatic Brain Injury in Rats. Journal of Neurotrauma, 36. doi:10.1089/neu.2019.6470

McAllister, T. W. (1992). Neuropsychiatry sequelae of head injuries. Psychiatric Clinics of North America, 15(2), 395-413. doi:doi:10.1016/s0193-953x(18)30245-4

McDougall, A., Bayley, M., \& Munce, S. E. (2018). The ketogenic diet as a treatment for traumatic brain injury: a scoping review. Brain Inj, 32(4), 416-422. doi:10.1080/02699052.2018.1429025

McHugh, L., \& Wood, R. (2008). Using a temporal discounting paradigm to measure decisionmaking and impulsivity following traumatic brain injury: A pilot study. Brain injury: [BI], 22, 715-721. doi:10.1080/02699050802263027

Medzhitov, R. (2008). Origin and physiological roles of inflammation. Nature, 454(7203), 428435. doi:10.1038/nature07201

Menon, D., Schwab, K., Wright, D., \& Maas, A. (2010). Position Statement: Definition of Traumatic Brain Injury. Archives of physical medicine and rehabilitation, 91, 1637-1640. doi:10.1016/j.apmr.2010.05.017 
Miller, A. A., \& Spencer, S. J. (2014). Obesity and neuroinflammation: a pathway to cognitive impairment. Brain Behav Immun, 42, 10-21. doi:10.1016/j.bbi.2014.04.001

Miller, E., \& Wallis, J. (2009). Executive function and higher-order cognition: definition and neural substrates. Encyclopedia of neuroscience, 4(99-104).

Mooney, G., \& Speed, J. (2001). The association between mild traumatic brain injury and psychiatric conditions. Brain Inj, 15(10), 865-877. doi:10.1080/02699050110065286

Moreno-Navarrete, J. M., Blasco, G., Puig, J., Biarnés, C., Rivero, M., Gich, J., . . FernándezReal, J. M. (2017). Neuroinflammation in obesity: circulating lipopolysaccharide-binding protein associates with brain structure and cognitive performance. Int J Obes (Lond), 41(11), 1627-1635. doi:10.1038/ijo.2017.162

Moreno-Navarrete, J. M., Ortega, F., Serino, M., Luche, E., Waget, A., Pardo, G., . . FernándezReal, J. M. (2012). Circulating lipopolysaccharide-binding protein (LBP) as a marker of obesity-related insulin resistance. Int J Obes (Lond), 36(11), 1442-1449. doi:10.1038/ijo.2011.256

Muccigrosso, M. M., Ford, J., Benner, B., Moussa, D., Burnsides, C., Fenn, A. M., . . Godbout, J. P. (2016). Cognitive deficits develop 1month after diffuse brain injury and are exaggerated by microglia-associated reactivity to peripheral immune challenge. Brain Behav Immun, 54, 95-109. doi:10.1016/j.bbi.2016.01.009

Mychasiuk, R., Hehar, H., \& Esser, M. J. (2015). A mild traumatic brain injury (mTBI) induces secondary attention-deficit hyperactivity disorder-like symptomology in young rats. Behav Brain Res, 286, 285-292. doi:10.1016/j.bbr.2015.03.010

Mychasiuk, R., Hehar, H., Ma, I., \& Esser, M. J. (2015). Dietary intake alters behavioral recovery and gene expression profiles in the brain of juvenile rats that have experienced a concussion. Frontiers in Behavioral Neuroscience, 9(17). doi:10.3389/fnbeh.2015.00017

Najjar, S., Pearlman, D. M., Alper, K., Najjar, A., \& Devinsky, O. (2013). Neuroinflammation and psychiatric illness. J Neuroinflammation, 10, 43. doi:10.1186/1742-2094-10-43

Nativ, A., Lazarus, J. A., Nativ, J., \& Joseph, J. (1994). Potentials associated with the Go/No-Go paradigm in traumatic brain injury. Arch Phys Med Rehabil, 75(12), 1322-1326.

Osier, N., \& Dixon, C. E. (2016). The Controlled Cortical Impact Model of Experimental Brain Trauma: Overview, Research Applications, and Protocol. Methods Mol Biol, 1462, 177 192. doi:10.1007/978-1-4939-3816-2_11

Ozga, J. E., Povroznik, J. M., Engler-Chiurazzi, E. B., \& Vonder Haar, C. (2018). Executive (dys)function after traumatic brain injury: special considerations for behavioral pharmacology. Behav Pharmacol, 29(7), 617-637. doi:10.1097/fbp.0000000000000430

Paxinos, G., \& Watson, C. (1982). The Rat Brain in Stereotaxic Coordinates (Seventh ed.): Elsevier Inc.

Pechey, R., \& Monsivais, P. (2016). Socioeconomic inequalities in the healthiness of food choices: Exploring the contributions of food expenditures. Prev Med, 88, 203-209. doi:10.1016/j.ypmed.2016.04.012

Pepping, J. K., Freeman, L. R., Gupta, S., Keller, J. N., \& Bruce-Keller, A. J. (2013). NOX2 deficiency attenuates markers of adiposopathy and brain injury induced by high-fat diet. Am J Physiol Endocrinol Metab, 304(4), E392-404. doi:10.1152/ajpendo.00398.2012

Prodam, F., Gasco, V., Caputo, M., Zavattaro, M., Pagano, L., Marzullo, P., . . . Aimaretti, G. (2013). Metabolic alterations in patients who develop traumatic brain injury (TBI)induced hypopituitarism. Growth Horm IGF Res, 23(4), 109-113. doi:10.1016/j.ghir.2013.04.001 
Rachlin, H., Raineri, A., \& Cross, D. (1991). Subjective probability and delay. J Exp Anal Behav, 55(2), 233-244. doi:10.1901/jeab.1991.55-233

Radler, M. E., Hale, M. W., \& Kent, S. (2014). Calorie restriction attenuates lipopolysaccharide (LPS)-induced microglial activation in discrete regions of the hypothalamus and the subfornical organ. Brain Behav Immun, 38, 13-24. doi:10.1016/j.bbi.2013.11.014

Ramlackhansingh, A. F., Brooks, D. J., Greenwood, R. J., Bose, S. K., Turkheimer, F. E., Kinnunen, K. M., . . . Sharp, D. J. (2011). Inflammation after trauma: microglial activation and traumatic brain injury. Ann Neurol, 70(3), 374-383. doi:10.1002/ana.22455

Rich, N. J., Van Landingham, J. W., Figueiroa, S., Seth, R., Corniola, R. S., \& Levenson, C. W. (2010). Chronic caloric restriction reduces tissue damage and improves spatial memory in a rat model of traumatic brain injury. J Neurosci Res, 88(13), 2933-2939. doi:10.1002/jnr.22443

Rochat, L., Beni, C., Annoni, J. M., Vuadens, P., \& Van der Linden, M. (2013). How inhibition relates to impulsivity after moderate to severe traumatic brain injury. $J$ Int Neuropsychol Soc, 19(8), 890-898. doi:10.1017/s1355617713000672

Rung, J. M., \& Madden, G. J. (2018). Experimental reductions of delay discounting and impulsive choice: A systematic review and meta-analysis. J Exp Psychol Gen, 147(9), 1349-1381. doi:10.1037/xge0000462

Salberg, S., Weerwardhena, H., Collins, R., Reimer, R. A., \& Mychasiuk, R. (2019). The behavioural and pathophysiological effects of the ketogenic diet on mild traumatic brain injury in adolescent rats. Behav Brain Res, 376, 112225. doi:10.1016/j.bbr.2019.112225

Shahar, D., Shai, I., Vardi, H., Shahar, A., \& Fraser, D. (2005). Diet and eating habits in high and low socioeconomic groups. Nutrition, 21(5), 559-566. doi:10.1016/j.nut.2004.09.018

Sharma, S., Fernandes, M. F., \& Fulton, S. (2013). Adaptations in brain reward circuitry underlie palatable food cravings and anxiety induced by high-fat diet withdrawal. Int J Obes (Lond), 37(9), 1183-1191. doi:10.1038/ijo.2012.197

Shaver, T. K., Ozga, J. E., Zhu, B., Anderson, K. G., Martens, K. M., \& Vonder Haar, C. (2019). Long-term deficits in risky decision-making after traumatic brain injury on a rat analog of the Iowa gambling task. Brain Res, 1704, 103-113. doi:10.1016/j.brainres.2018.10.004

Shimakawa, T., Sorlie, P., Carpenter, M. A., Dennis, B., Tell, G. S., Watson, R., \& Williams, O. D. (1994). Dietary intake patterns and sociodemographic factors in the atherosclerosis risk in communities study. ARIC Study Investigators. Prev Med, 23(6), 769-780. doi:10.1006/pmed.1994.1133

Shultz, S. R., Bao, F., Omana, V., Chiu, C., Brown, A., \& Cain, D. P. (2012). Repeated mild lateral fluid percussion brain injury in the rat causes cumulative long-term behavioral impairments, neuroinflammation, and cortical loss in an animal model of repeated concussion. J Neurotrauma, 29(2), 281-294. doi:10.1089/neu.2011.2123

Simon, D. W., McGeachy, M. J., Bayır, H., Clark, R. S., Loane, D. J., \& Kochanek, P. M. (2017). The far-reaching scope of neuroinflammation after traumatic brain injury. Nat Rev Neurol, 13(3), 171-191. doi:10.1038/nrneurol.2017.13

Smith, B. L., Schmeltzer, S. N., Packard, B. A., Sah, R., \& Herman, J. P. (2016). Divergent effects of repeated restraint versus chronic variable stress on prefrontal cortical immune status after LPS injection. Brain Behav Immun, 57, 263-270.

doi:10.1016/j.bbi.2016.05.004 
Smith, J. A., Das, A., Ray, S. K., \& Banik, N. L. (2012). Role of pro-inflammatory cytokines released from microglia in neurodegenerative diseases. Brain Res Bull, 87(1), 10-20. doi:10.1016/j.brainresbull.2011.10.004

Steele, C. C., Pirkle, J. R. A., Davis, I. R., \& Kirkpatrick, K. (2019). Dietary effects on the determinants of food choice: Impulsive choice, discrimination, incentive motivation, preference, and liking in male rats. Appetite, 136, 160-172. doi:10.1016/j.appet.2019.01.023

Steele, C. C., Pirkle, J. R. A., \& Kirkpatrick, K. (2017). Diet-induced impulsivity: Effects of a high-fat and a high-sugar diet on impulsive choice in rats. PLoS One, 12(6), e0180510. doi:10.1371/journal.pone.0180510

Tang, Y., \& Le, W. (2016). Differential Roles of M1 and M2 Microglia in Neurodegenerative Diseases. Molecular Neurobiology, 53(2), 1181-1194. doi:10.1007/s12035-014-9070-5

Taylor, A. N., Tio, D. L., Paydar, A., \& Sutton, R. L. (2018). Sex Differences in Thermal, Stress, and Inflammatory Responses to Minocycline Administration in Rats with Traumatic Brain Injury. J Neurotrauma, 35(4), 630-638. doi:10.1089/neu.2017.5238

Tikka, T., Fiebich, B. L., Goldsteins, G., Keinanen, R., \& Koistinaho, J. (2001). Minocycline, a tetracycline derivative, is neuroprotective against excitotoxicity by inhibiting activation and proliferation of microglia. J Neurosci, 21(8), 2580-2588. doi:10.1523/jneurosci.2108-02580.2001

Urbain, P., Strom, L., Morawski, L., Wehrle, A., Deibert, P., \& Bertz, H. (2017). Impact of a 6week non-energy-restricted ketogenic diet on physical fitness, body composition and biochemical parameters in healthy adults. Nutr Metab (Lond), 14, 17. doi:10.1186/s12986-017-0175-5

Vaishnavi, S., Rao, V., \& Fann, J. R. (2009). Neuropsychiatric problems after traumatic brain injury: unraveling the silent epidemic. Psychosomatics, 50(3), 198-205. doi:10.1176/appi.psy.50.3.198

van den Bos, R., Koot, S., \& de Visser, L. (2014). A rodent version of the Iowa Gambling Task: 7 years of progress. Front Psychol, 5, 203. doi:10.3389/fpsyg.2014.00203

van den Bos, R., Lasthuis, W., den Heijer, E., van der Harst, J., \& Spruijt, B. (2006). Toward a rodent model of the Iowa gambling task. Behav Res Methods, 38(3), 470-478. doi:10.3758/bf03192801

Vonder Haar, C., Anderson, G. D., \& Hoane, M. R. (2011). Continuous nicotinamide administration improves behavioral recovery and reduces lesion size following bilateral frontal controlled cortical impact injury. Behav Brain Res, 224(2), 311-317. doi:10.1016/j.bbr.2011.06.009

Vonder Haar, C., Lam, F. C., Adams, W. K., Riparip, L. K., Kaur, S., Muthukrishna, M., . . . Winstanley, C. A. (2016). Frontal Traumatic Brain Injury in Rats Causes Long-Lasting Impairments in Impulse Control That Are Differentially Sensitive to Pharmacotherapeutics and Associated with Chronic Neuroinflammation. ACS Chem Neurosci, 7(11), 1531-1542. doi:10.1021/acschemneuro.6b00166

Vonder Haar, C., Martens, K. M., Riparip, L. K., Rosi, S., Wellington, C. L., \& Winstanley, C. A. (2017). Frontal Traumatic Brain Injury Increases Impulsive Decision Making in Rats: A Potential Role for the Inflammatory Cytokine Interleukin-12. J Neurotrauma, 34(19), 2790-2800. doi:10.1089/neu.2016.4813

Vormann, J. (2003). Magnesium: nutrition and metabolism. Mol Aspects Med, 24(1-3), 27-37. doi:10.1016/s0098-2997(02)00089-4 
Waters-Wood, S. M., Xiao, L., Denburg, N. L., Hernandez, M., \& Bechara, A. (2012). Failure to learn from repeated mistakes: persistent decision-making impairment as measured by the iowa gambling task in patients with ventromedial prefrontal cortex lesions. $J$ Int Neuropsychol Soc, 18(5), 927-930. doi:10.1017/s135561771200063x

Weng, L., Dong, S., Wang, S., Yi, L., \& Geng, D. (2019). Macranthol attenuates lipopolysaccharide-induced depressive-like behaviors by inhibiting neuroinflammation in prefrontal cortex. Physiol Behav, 204, 33-40. doi:10.1016/j.physbeh.2019.02.010

Whelan-Goodinson, R., Ponsford, J. L., Schönberger, M., \& Johnston, L. (2010). Predictors of psychiatric disorders following traumatic brain injury. J Head Trauma Rehabil, 25(5), 320-329. doi:10.1097/HTR.0b013e3181c8f8e7

Winstanley, C. A., Eagle, D. M., \& Robbins, T. W. (2006). Behavioral models of impulsivity in relation to ADHD: translation between clinical and preclinical studies. Clin Psychol Rev, 26(4), 379-395. doi:10.1016/j.cpr.2006.01.001

Xiong, Y., Zhang, Y., Mahmood, A., Meng, Y., Zhang, Z. G., Morris, D. C., \& Chopp, M. (2012). Neuroprotective and neurorestorative effects of thymosin $\beta 4$ treatment initiated 6 hours after traumatic brain injury in rats. J Neurosurg, 116(5), 1081-1092. doi:10.3171/2012.1.Jns111729

Yeates, K. O., Swift, E., Taylor, H. G., Wade, S. L., Drotar, D., Stancin, T., \& Minich, N. (2004). Short- and long-term social outcomes following pediatric traumatic brain injury. J Int Neuropsychol Soc, 10(3), 412-426. doi:10.1017/s1355617704103093

Yeates, K. O., Taylor, H. G., Drotar, D., Wade, S. L., Klein, S., Stancin, T., \& Schatschneider, C. (1997). Preinjury family environment as a determinant of recovery from traumatic brain injuries in school-age children. J Int Neuropsychol Soc, 3(6), 617-630.

Zeeb, F. D., \& Winstanley, C. A. (2013). Functional disconnection of the orbitofrontal cortex and basolateral amygdala impairs acquisition of a rat gambling task and disrupts animals' ability to alter decision-making behavior after reinforcer devaluation. J Neurosci, 33(15), 6434-6443. doi:10.1523/jneurosci.3971-12.2013

Zgaljardic, D. J., Seale, G. S., Schaefer, L. A., Temple, R. O., Foreman, J., \& Elliott, T. R. (2015). Psychiatric Disease and Post-Acute Traumatic Brain Injury. Journal of Neurotrauma, 32(23), 1911-1925. doi:10.1089/neu.2014.3569

Ziablitsev, S. V., Pishchulina, S. V., Kolesnikova, S. V., Boris, R. N., \& Yuzkiv Ya, S. (2016). Disorders of carbohydrate metabolism in experimental brain injury. Fiziol Zh, 62(4), 1822. doi:10.15407/fz62.04.018

Zinchenko, O., \& Enikolopova, E. (2017). The Impact of Executive Functions and Emotional Intelligence on Iowa Gambling Task Performance: Focus on Right Frontal Lobe Damage. Arch Clin Neuropsychol, 32(8), 1026-1036. doi:10.1093/arclin/acx065

Zucker, I., \& Beery, A. K. (2010). Males still dominate animal studies. Nature, 465(7299), 690. doi:10.1038/465690a 